https://helda.helsinki.fi

\title{
Limits of Sobolev homeomorphisms
}

\section{Iwaniec, Tadeusz}

2017

Iwaniec , T \& Onninen , J 2017 , ' Limits of Sobolev homeomorphisms ' , European

Mathematical Society. Journal, vol. 19 , no. 2 , pp. 473-505 . https://doi.org/10.4171/JEMS/671

http://hdl.handle.net/10138/312802

https://doi.org/10.4171/JEMS/671

publishedVersion

Downloaded from Helda, University of Helsinki institutional repository.

This is an electronic reprint of the original article.

This reprint may differ from the original in pagination and typographic detail.

Please cite the original version. 
DOI 10.4171/JEMS/671

Tadeusz Iwaniec · Jani Onninen

\section{Limits of Sobolev homeomorphisms}

Received January 21, 2014 and in revised form November 29, 2014

Abstract. Let $\mathbb{X}, \mathbb{Y} \subset \mathbb{R}^{2}$ be topologically equivalent bounded Lipschitz domains. We prove that weak and strong limits of homeomorphisms $h: \mathbb{X} \stackrel{\text { onto }}{\longrightarrow} \mathbb{Y}$ in the Sobolev space $\mathscr{W}^{1, p}\left(\mathbb{X}, \mathbb{R}^{2}\right)$, $p \geq 2$, are the same. As an application, we establish the existence of 2D-traction free minimal deformations for fairly general energy integrals.

Keywords. Energy-minimal deformations, approximation of Sobolev homeomorphisms, variational integrals, harmonic mappings, $p$-harmonic equation

\section{Introduction}

Throughout this text, $\mathbb{X}$ and $\mathbb{Y}$ are bounded Lipschitz domains in $\mathbb{R}^{2} \simeq \mathbb{C}$ of the same topological type, and $\mathscr{H}(\mathbb{X}, \mathbb{Y})$ denotes the set of all orientation preserving homeomorphisms $h: \mathbb{X} \stackrel{\text { onto }}{\longrightarrow} \mathbb{Y}$. Our interest is in the subspace $\mathscr{H}_{p}(\mathbb{X}, \mathbb{Y}):=\mathscr{H}(\mathbb{X}, \mathbb{Y}) \cap$ $\mathscr{W}^{1, p}\left(\mathbb{X}, \mathbb{R}^{2}\right), p \geq 2$.

Remark 1.1. The results we are about to demonstrate also hold for domains with isolated points as boundary components. However, such generality gives essentially nothing better than the case of Lipschitz domains. This is because every homeomorphism $h \in \mathscr{W}^{1, p}\left(\mathbb{X}, \mathbb{R}^{2}\right), p \geq 2$, of a domain with a singleton $\left\{x_{\circ}\right\}$ as a boundary component extends to $\mathbb{X} \cup\left\{x_{\circ}\right\}$ as a homeomorphism in the Sobolev space $\mathscr{W}^{1, p}\left(\mathbb{X} \cup\left\{x_{\circ}\right\}, \mathbb{R}^{2}\right)$. That is why we may, and do, assume that each boundary $\partial \mathbb{X}$ and $\partial \mathbb{Y}$ consists of the same number, say $1 \leq \ell<\infty$, of Lipschitz disjoint closed Jordan curves. We say that the domains are multiply connected if $\ell \geq 2$, and simply connected if $\ell=1$. Let $\mathscr{W}_{0}^{1, p}\left(\mathbb{X}, \mathbb{R}^{2}\right)$ denote the completion of $\mathscr{C}_{\circ}^{\infty}\left(\mathbb{X}, \mathbb{R}^{2}\right)$ in $\mathscr{W}^{1, p}\left(\mathbb{X}, \mathbb{R}^{2}\right)$.

T. Iwaniec: Department of Mathematics, Syracuse University, Syracuse, NY 13244, USA, and Department of Mathematics and Statistics, University of Helsinki, Finland; e-mail: tiwaniec@syr.edu

J. Onninen: Department of Mathematics, Syracuse University, Syracuse, NY 13244, USA, and Department of Mathematics and Statistics, P.O. Box 35, FI- 40014, University of Jyväskylä, Finland; e-mail: jkonnine@ syr.edu

Mathematics Subject Classification (2010): Primary 30E10; Secondary 46E35, 58E20 


\subsection{Main result}

The chief conclusion of this paper is that weak and strong limits of Sobolev homeomorphisms are the same thing.

Theorem 1.2. Let $\mathbb{X}$ and $\mathbb{Y}$ be bounded multiply connected Lipschitz domains in $\mathbb{R}^{2}$ and $h_{j}: \mathbb{X} \stackrel{\text { onto }}{\longrightarrow} \mathbb{Y}$ homeomorphisms converging weakly to $h$ in the space $\mathscr{W}^{1, p}\left(\mathbb{X}, \mathbb{R}^{2}\right), p \geq 2$. Then there exists a sequence of homeomorphisms (actually $\mathscr{C}^{\infty}$-diffeomorphisms)

$$
h_{j}^{*}: \mathbb{X} \stackrel{\text { onto }}{\longrightarrow} \mathbb{Y}, \quad h_{j}^{*} \in h+\mathscr{W}_{\circ}^{1, p}\left(\mathbb{X}, \mathbb{R}^{2}\right),
$$

converging to $h$ strongly in $\mathscr{W}^{1, p}\left(\mathbb{X}, \mathbb{R}^{2}\right)$. The same holds for simply connected domains, except for $p=2$, in which case the mappings $h_{j}$ must be fixed either at one point in $\mathbb{X}$ or at three points on $\partial \mathbb{X}$.

For this last case, the reader may wish to consider a sequence of conformal mappings of the unit disk onto itself, which take the center of the disk into points approaching its boundary. The weak limit, being a constant map, cannot be strongly approximated by homeomorphisms in $\mathscr{W}^{1, p}\left(\mathbb{X}, \mathbb{R}^{2}\right), p \geq 2$.

Weak limits of Sobolev homeomorphisms arise naturally (as energy-minimal mappings) in both Geometric Function Theory (GFT) [5, 34, 63] and Nonlinear Elasticity (NE) $[4,8,18,55,64,67]$. It should be noted that in general weak and strong limits of homeomorphisms $\mathbb{X} \stackrel{\text { onto }}{\longrightarrow} \mathbb{Y}$ take $\mathbb{X}$ into the closure of $\mathbb{Y}$. Nevertheless, by convenient abuse of customary notation, denote by $\overline{\mathscr{H}}_{p}(\mathbb{X}, \mathbb{Y})$ and $\widetilde{\mathscr{H}}_{p}(\mathbb{X}, \mathbb{Y})$ the classes of strong and weak limits of homeomorphisms in $\mathscr{H}_{p}(\mathbb{X}, \mathbb{Y})$, respectively. Theorem 1.2 tells us that

$$
\mathscr{H}_{p}(\mathbb{X}, \mathbb{Y}) \subset \overline{\mathscr{H}_{p}}(\mathbb{X}, \mathbb{Y})=\widetilde{\mathscr{H}}_{p}(\mathbb{X}, \mathbb{Y}) \subset \mathscr{W}^{1, p}\left(\mathbb{X}, \mathbb{R}^{2}\right)
$$

Remark 1.3. Further improvements on homeomorphisms $h_{j}^{*}$ in Theorem 1.2 are possible. First, in view of [30], each homeomorphism $h_{j}^{*}: \mathbb{X} \stackrel{\text { onto }}{\longrightarrow} \mathbb{Y}$ can be strongly approximated in $\mathscr{W}^{1, p}(\mathbb{X}, \mathbb{Y})$ by $\mathscr{C}^{\infty}$-diffeomorphisms which agree with $h_{j}^{*}$ on $\partial \mathbb{X}$. That is why one can take $\mathscr{C}^{\infty}$-diffeomorphisms for $h_{j}^{*}$ in (1.1). For $p=2$, other advances come from recent results in [31]. Accordingly, one may find diffeomorphisms $h_{j}^{*}: \mathbb{X} \stackrel{\text { onto }}{\longrightarrow} \mathbb{Y}$ (strongly converging to $h$ ) which extend as homeomorphisms between the closures of $\mathbb{X}$ and $\mathbb{Y}$, obviously at the sacrifice of the boundary conditions in (1.1).

For the proof of Theorem 1.2 the natural idea of correcting mappings $\left\{h_{j}\right\}$ to gain strong convergence does not work. Instead, we construct the sequence $\left\{h_{j}^{*}\right\}$ through a series of local replacements of the limit mapping $h$ with $p$-harmonic diffeomorphisms. At this stage we take advantage of the celebrated Radó-Kneser-Choquet Theorem [23] and its generalization to the $p$-harmonic setting due to Alessandrini and Sigalotti [3]. We employ quasiconformal mappings so that the associated complex Beltrami type equations come into play. A result of independent interest is a $p$-harmonic variant of the Hurwitz Theorem (Theorem 4.13). 


\subsection{Weak (sequential) closure versus strong closure of Sobolev homeomorphisms}

As is well known, in any Banach space weakly closed convex sets and closed convex sets are the same. Mazur's Lemma tells us that to every weakly converging sequence there corresponds a sequence of convex combinations of its members that converges strongly to the same limit. However, for nonconvex sets (such as our sets of homeomorphisms) the relations between sequential weak closure and strong closure are only partially understood. In general, the set of all weak limits of sequences assembled from a subset of a Banach space need not be sequentially weakly closed. Let $\widehat{\mathscr{H}}_{p}(\mathbb{X}, \mathbb{Y})$ denote the sequential weak closure of $\mathscr{H}_{p}(\mathbb{X}, \mathbb{Y})$. Recall the class $\widetilde{\mathscr{H}}_{p}(\mathbb{X}, \mathbb{Y})$ of weak limits of homeomorphisms in $\mathscr{H}_{p}(\mathbb{X}, \mathbb{Y})$. By virtue of Theorem 1.2 , it is a simple matter to see that $\widehat{\mathscr{H}}_{p}(\mathbb{X}, \mathbb{Y})$ actually coincides with the strong closure.

Corollary 1.4. Let $\mathbb{X}$ and $\mathbb{Y}$ be bounded multiply connected Lipschitz domains in $\mathbb{R}^{2}$ and $2 \leq p<\infty$. Then

$$
\widehat{\mathscr{H}}_{p}(\mathbb{X}, \mathbb{Y})=\widetilde{\mathscr{H}_{p}}(\mathbb{X}, \mathbb{Y})=\overline{\mathscr{H}_{p}}(\mathbb{X}, \mathbb{Y})
$$

\section{The existence of 2D-traction free minimal deformations}

We can now pose problems whose solutions demonstrate how Theorem 1.2 can be used for the variational approach to Geometric Function Theory (GFT), with relevance to Nonlinear Elasticity (NE); specifically, energy-minimal deformations of thin plates (2Ddomains).

Let us commence with NE. The $2 \times 2$ differential matrix $D h(x) \in \mathbb{R}^{2 \times 2}$, called the deformation gradient, is well defined almost everywhere in $\mathbb{X}$. It is axiomatic that the hyperelastic deformations $h: \mathbb{X} \stackrel{\text { onto }}{\longrightarrow} \mathbb{Y}$ are the minima of a given energy integral

$$
\mathscr{E}[h]=\int_{\mathbb{X}} \mathbf{E}(x, h, D h) d x
$$

The integrand $\mathbf{E}: \mathbb{X} \times \mathbb{Y} \times \mathbb{R}^{2 \times 2} \rightarrow \mathbb{R}$ (stored energy function) tells us something about the elastic features of the materials in $\mathbb{X}$ and $\mathbb{Y}$. Nowadays, advanced mathematical models deal with stored energy functions that satisfy Morrey's quasiconvexity condition with respect to the deformation gradient [59]. Without going into details, a consequence of quasiconvexity is lower semicontinuity. Let us specify this property for our purpose as follows:

$$
\mathscr{E}_{\mathbb{X}}[h] \leq \liminf \mathscr{E}_{\mathbb{X}}\left[h_{j}\right] \quad \text { whenever } h_{j} \in \mathscr{H}_{p}(\mathbb{X}, \mathbb{Y}) \text { converge weakly to } h
$$

We shall further impose on $\mathbf{E}$ the following coercivity condition:

$$
\int_{\mathbb{X}}|D h(x)|^{p} d x \leq C \int_{\mathbb{X}} \mathbf{E}(x, h, D h) d x \quad \text { for all } h \in \mathscr{H}_{p}(\mathbb{X}, \mathbb{Y}) .
$$


And we assume continuity of the energy integral with respect to strong convergence. More precisely, whenever mappings $h_{j} \in \mathscr{H}_{p}(\mathbb{X}, \mathbb{Y})$ converge to $h$ in the norm topology of $\mathscr{W}^{1, p}\left(\mathbb{X}, \mathbb{R}^{2}\right)$, we have

$$
\mathscr{E} \mathbb{X}[h]=\liminf \mathscr{E} \mathbb{X}\left[h_{j}\right]
$$

Verification of (2.2)-(2.4) presents no difficulty in widely studied energy-minimal mappings.

In GFT, on the other hand, to every quasiconformal mapping $f: \mathbb{X} \stackrel{\text { onto }}{\longrightarrow} \mathbb{Y}$ there corresponds a distinctive conformal energy for which $f$ is an absolute minimizer, that is, with smallest energy among all homeomorphisms $f: \mathbb{X} \stackrel{\text { onto }}{\longrightarrow} \mathbb{Y}$. Note that in this minimization problem we allow $f$ to slide along the boundaries, no boundary values are prescribed upfront. We call it the traction free minimization problem. As an example, consider a quasilinear complex Beltrami equation for a $K$-quasiconformal map $f \in \mathscr{H}_{2}(\mathbb{X}, \mathbb{Y})$,

$$
\frac{\partial f}{\partial \bar{z}}=\mu(z, f) \frac{\partial f}{\partial z}, \quad|\mu| \leq k:=\frac{K-1}{K+1}<1 \quad \text { in } \mathbb{X} \times \mathbb{Y} .
$$

Here the function $\mu: \mathbb{X} \times \mathbb{Y} \rightarrow \mathbb{C}$ (a complex Beltrami coefficient) comes naturally from specific measurable Riemannian metric tensors on $\mathbb{X}$ and $\mathbb{Y}$. With respect to those metrics, $f$ becomes a conformal transformation. In this setting the relevant energy integral, defined for all homeomorphisms $h \in \mathscr{H}_{2}(\mathbb{X}, \mathbb{Y})$, is the following (note it reduces to the Dirichlet energy if $\mu \equiv 0$ ):

$$
\begin{aligned}
\mathscr{E}[h] & =\int_{\mathbb{X}} \frac{\left|h_{z}-\bar{\mu} h_{\bar{z}}\right|^{2}+\left|h_{\bar{z}}-\mu h_{z}\right|^{2}}{1-|\mu(z, h)|^{2}} d z=\int_{\mathbb{X}}\left(\frac{2\left|h_{\bar{z}}-\mu h_{z}\right|^{2}}{1-|\mu(z, h)|^{2}}+\left|h_{z}\right|^{2}-\left|h_{\bar{z}}\right|^{2}\right) d z \\
& \geq \int_{\mathbb{X}}\left(\left|h_{z}\right|^{2}-\left|h_{\bar{z}}\right|^{2}\right) d z=|\mathbb{Y}| .
\end{aligned}
$$

In the above energy estimate equality occurs iff $h$ satisfies the first order complex Beltrami system (2.5). An additional interest in the energy functional (2.6) arises when the first order Beltrami equation (2.5) admits no homeomorphic solution $h: \mathbb{X} \stackrel{\text { onto }}{\longrightarrow} \mathbb{Y}$, which is typical for multiply connected domains [5, 6]. Consequently, the lower bound (2.6) among all homeomorphisms in $\mathscr{H}_{2}(\mathbb{X}, \mathbb{Y})$ is larger than $|\mathbb{Y}|$. New, sometimes unexpected, phenomena of interpenetration of matter occur, stimulating further studies of such energies. The existence of energy-minimal mappings (injective or not) is the vital part of these new studies. Nowadays the Quasiconformal Mapping Theory is already enriched by letting in energy-minimal deformations [21, 29, 38, 44, 45, 46] which, like complex harmonic mappings, are solutions of second order Lagrange equations rather than first order Beltrami type systems. The traction free solutions can be realized physically, for example as deformations of incompressible material confined in a given box $[8,9,10]-\mathrm{a}$ new connection with NE.

\subsection{Existence of traction free minimal deformations (no Lavrent'ev's phenomenon)}

It is certainly unrealistic to expect that the infimum energy will be attained within Sobolev homeomorphisms $h: \mathbb{X} \stackrel{\text { onto }}{\longrightarrow} \mathbb{Y}$. The best illustration is the collapse of injectivity in the Dirichlet energy-minimal map between circular annuli [6, 40] (see also [41] for more 
examples). Except for a few specific cases [37] the collapse of injectivity remains an open question for intensively studied neohookean energy functionals [11, 15, 20, 24, 65].

This suggests a more general point of view. Let us take a quick look at the energy functional

$$
\mathscr{E}_{\mathbb{X}}[h]=\int_{\mathbb{X}} \mathbf{E}(x, h, D h) d x \quad \text { defined for } h \in \mathscr{H}_{p}(\mathbb{X}, \mathbb{Y}),
$$

with the purpose of determining its infimum value,

$$
\mathrm{E}(\mathbb{X}, \mathbb{Y}):=\inf _{h \in \mathscr{H}_{p}(\mathbb{X}, \mathbb{Y})} \iint_{\mathbb{X}} \mathbf{E}(x, h, D h) d x
$$

We address the following question:

Question 2.1. What is the smallest subspace $\mathscr{F}(\mathbb{X}, \mathbb{Y}) \subset W^{1, p}\left(\mathbb{X}, \mathbb{R}^{n}\right)$, containing homeomorphisms in $H_{p}(\mathbb{X}, \mathbb{Y})$, within which the minimum energy is attained, and furthermore

$$
\min _{h \in \mathscr{F}(\mathbb{X}, \mathbb{Y})} \int_{\mathbb{X}} \mathbf{E}(x, h, D h) d x=\inf _{h \in \mathscr{H}_{p}(\mathbb{X}, \mathbb{Y})} \int_{\mathbb{X}} \mathbf{E}(x, h, D h) d x ?
$$

That is to say, we wish to avoid Lavrent'ev's phenomenon in finding energy-minimal deformations.

By virtue of Theorem 1.2 the subspace $\mathscr{F}(\mathbb{X}, \mathbb{Y})=\overline{\mathscr{H}_{p}(\mathbb{X}, \mathbb{Y})}$ serves this purpose perfectly. Indeed, we have

Theorem 2.2. Let $\mathbb{X}, \mathbb{Y} \subset \mathbb{R}^{2}$ be bounded multiply connected Lipschitz domains of the same topological type. Consider the energy integral

$$
\mathscr{E}[h]=\int_{\mathbb{X}} \mathbf{E}(x, h, D h) d x \quad \text { defined for } h \in \overline{\mathscr{H}_{p}}(\mathbb{X}, \mathbb{Y}), p \geq 2
$$

Then, under the conditions (2.2)-(2.4), there exists $h_{\circ} \in \overline{\mathscr{H}_{p}}(\mathbb{X}, \mathbb{Y})$ such that

$$
\mathscr{E}\left[h_{\circ}\right]=\inf _{h \in \mathscr{H}_{p}(\mathbb{X}, \mathbb{Y})} \int_{\mathbb{X}} \mathbf{E}(x, h, D h) d x .
$$

Some specific examples have already been known [6, 32, 37, 40, 41].

\subsection{Partially traction free problems}

It should be noted that homeomorphisms $h: \mathbb{X} \stackrel{\text { onto }}{\longrightarrow} \mathbb{Y}$ (between Lipschitz domains) in the Sobolev space $\mathscr{W}^{1, p}\left(\mathbb{X}, \mathbb{R}^{2}\right), p \geq 2$, and their weak limits extend continuously as monotone mappings between the closures, still denoted by $h: \overline{\mathbb{X}} \rightarrow \overline{\mathbb{Y}}$. Monotonicity, the concept of C. B. Morrey [58], means that the preimage $h^{-1}(C)$ of a continuum $C \subset \overline{\mathbb{Y}}$ is a continuum in $\overline{\mathbb{X}}$. The modulus of continuity is controlled by the $\mathscr{L}^{p}$-norm of the 
gradient-see [39] for such results. Note that therefore a $\mathscr{W}^{1, p}$-weakly converging sequence of homeomorphisms between $\mathbb{X}$ and $\mathbb{Y}$ actually converges uniformly. Now, we choose and fix a mapping $g \in \mathscr{H}_{p}(\mathbb{X}, \mathbb{Y})$ and a compact subset $K \subset \partial \mathbb{X}$, for example, a union of selected boundary components. This $g$ will serve as a boundary data for a partially traction free problem. Accordingly, we introduce the following admissible family:

$$
\mathscr{H}_{p}(\mathbb{X}, \mathbb{Y} ; g, K)=\left\{h \in \mathscr{H}_{p}(\mathbb{X}, \mathbb{Y}): h_{\mid K}=g_{\mid K}(\text { upon continuous extension to } \partial \mathbb{X})\right\}
$$

and denote its strong closure by $\overline{\mathscr{H}_{p}}(\mathbb{X}, \mathbb{Y} ; g, K) \subset \mathscr{W}^{1, p}\left(\mathbb{X}, \mathbb{R}^{2}\right)$. Actually, the strong closure coincides with the weak (sequential) closure. With the aid of Theorem 1.2, by the direct method in the calculus of variations, we obtain:

Theorem 2.3. Under the same hypotheses as in Theorem 2.2 there exists $h_{\circ} \in$ $\overline{\mathscr{H}_{p}}(\mathbb{X}, \mathbb{Y} ; g, K)$ such that

$$
\mathscr{E}\left[h_{\circ}\right]=\inf _{h \in \mathscr{H}_{p}(\mathbb{X}, \mathbb{Y} ; g, K)} \int_{\mathbb{X}} \mathbf{E}(x, h, D h) d x .
$$

\subsection{Remaks on monotone Sobolev mappings}

Uniform approximation of monotone mappings with homeomorphisms is of great interest in topology. We refer the interested reader to Youngs [71, 72] and Radó [61] for the early results and to [56, 70] for further developments. In the Sobolev setting, let $\mathbb{X} \subset \mathbb{R}^{2}$ and $\mathbb{Y} \subset \mathbb{R}^{2}$ be bounded Lipschitz domains of the same topological type. Then every continuous monotone map $h: \overline{\mathbb{X}} \stackrel{\text { onto }}{\longrightarrow} \overline{\mathbb{Y}}$ of Sobolev class $\mathscr{W}^{1, p}\left(\mathbb{X}, \mathbb{R}^{2}\right), p>1$, can be approximated uniformly and strongly in $\mathscr{W}^{1, p}\left(\mathbb{X}, \mathbb{R}^{2}\right.$ ) by homeomorphisms $h_{j}: \mathbb{X} \stackrel{\text { onto }}{\longrightarrow} \mathbb{Y}$ (and with diffeomorphisms as well). This result has been established by the present authors in [42]. The reader may wish to notice that for $1<p<2$ the lack of uniform continuity estimates prevents a $\mathscr{W}^{1, p}$-limit of homeomorphisms from being continuous and monotone. Nevertheless, if for some reason, a sequence of homeomorphisms $h_{j}: \mathbb{X} \stackrel{\text { onto }}{\longrightarrow} \mathbb{Y}$ converges uniformly and weakly in $\mathscr{W}^{1, p}(\mathbb{X}, \mathbb{Y}), 1 \leq p<2$, then its limit (being a monotone map) can also be approximated by diffeomorphisms $h_{j}: \mathbb{X} \stackrel{\text { onto }}{\longrightarrow} \mathbb{Y}$, uniformly and strongly in $\mathscr{W}^{1, p}(\mathbb{X}, \mathbb{Y})$.

Question 2.4. Does Theorem 1.2 also hold for all $1<p<2$, that is, without the additional assumption that the sequence $h_{j}: \mathbb{X} \stackrel{\text { onto }}{\longrightarrow} \mathbb{Y}$ converges uniformly?

\subsection{Practical significance of $\overline{\mathscr{H}_{p}}(\mathbb{X}, \mathbb{Y})$}

Theorems 2.2 and 2.3 assert that the minimum energy is always attained in the Sobolev class $\overline{\mathscr{H}}_{p}(\mathbb{X}, \mathbb{Y})$, that is, within the strong limits of homeomorphisms. This result is completely new in the theory of traction free deformations; and it is very useful when injectivity of the minimal map fails. In this case the energy-minimal map tells us that we must stop the minimizing sequence prior to the collapse of injectivity. This latter development can be interpreted as interpenetration of matter. Is there any better motivation for studying the class $\overline{\mathscr{H}_{p}}(\mathbb{X}, \mathbb{Y})$ in the theory of Nonlinear Elasticity? 
The rest of the paper is devoted to a thorough proof of Theorem 1.2. We worked out fairly detailed arguments. Several technical novelties cannot be dispensed with without compromising the completeness of the proofs-see for example Proposition 3.1 (not obvious at all) or Lemma 4.5 .

\section{Geometric preliminaries}

\subsection{Domains}

Recall that $\mathbb{X}$ and $\mathbb{Y}$ are bounded Lipschitz domains in $\mathbb{R}^{2} \simeq \mathbb{C}$ of connectivity $1 \leq \ell$ $<\infty$. Each boundary $\partial \mathbb{X}$ and $\partial \mathbb{Y}$ consists of $\ell$ disjoint closed Jordan curves which locally, upon suitable rotation, become graphs of Lipschitz functions. We reserve the ordered $\ell$-tuples

$$
\begin{array}{ll}
\left(\mathfrak{X}_{1}, \ldots, \mathfrak{X}_{\ell}\right) & \text { for the components of } \partial \mathbb{X}, \\
\left(\Upsilon_{1}, \ldots, \Upsilon_{\ell}\right) & \text { for the components of } \partial \mathbb{Y} .
\end{array}
$$

Let us emphasize that a bi-Lipschitz image of a smooth $\ell$-connected domain need not be Lipschitz [43, 68, 69]. For the converse, however, any Lipschitz domain can be mapped via a bi-Lipschitz transformation $\Psi: \mathbb{C} \stackrel{\text { onto }}{\longrightarrow} \mathbb{C}$ onto a domain whose boundaries are circles; we refer to it as a Schottky domain. We shall take advantage of such bi-Lipschitz transformations; they will simplify the forthcoming extension procedures and cause no loss of generality.

\subsection{Reduction to Schottky domains}

Transition from a Lipschitz domain $\mathbb{X}$ to a circular domain $\mathbb{X}^{\prime}$ via a bi-Lipschitz transformation $T: \mathbb{R}^{2} \stackrel{\text { onto }}{\longrightarrow} \mathbb{R}^{2}$ such that $T: \mathbb{X}^{\prime} \stackrel{\text { onto }}{\longrightarrow} \mathbb{X}$ presents no difficulty. For, every such transformation induces a composition operator

$$
T_{b}: \mathscr{W}^{1, p}(\mathbb{X}, \mathbb{Y}) \rightarrow \mathscr{W}^{1, p}\left(\mathbb{X}^{\prime}, \mathbb{Y}\right), \quad T_{b}(h)=g, \quad g:=h \circ T,
$$

which is a linear isomorphism. Surprisingly enough, an analogous transformation of the target domain $\mathbb{Y}$ takes some effort because the continuity (in the Sobolev norm) of the composition operator with a bi-Lipschitz transformation is not always the case.

Let $\mathbb{Y} \subset \mathbb{R}^{2}$ be a bounded domain and $F: \mathbb{Y} \stackrel{\text { onto }}{\longrightarrow} \mathbb{Y}^{\prime}$ a bi-Lipschitz transformation. Then, whatever the domain $\mathbb{X}$, the induced composition map

$$
F_{\sharp}: \mathscr{W}^{1, p}(\mathbb{X}, \mathbb{Y}) \rightarrow \mathscr{W}^{1, p}\left(\mathbb{X}, \mathbb{Y}^{\prime}\right), \quad F_{\sharp}(g):=F \circ g,
$$

is well defined as a bounded nonlinear operator, though its continuity is not always guaranteed [26]. There exists, however, a bi-Lipschitz transformation which works perfectly for our purposes.

Proposition 3.1. Given any bounded Lipschitz domain $\mathbb{Y} \subset \mathbb{R}^{2}$, there exists a bi-Lipschitz map $F: \mathbb{R}^{2} \stackrel{\text { onto }}{\longrightarrow} \mathbb{R}^{2}$ which takes $\mathbb{Y}$ onto a circular domain $\mathbb{Y}^{\prime}$. The induced composition operator $F_{\sharp}: \mathscr{W}^{1, p}(\mathbb{X}, \mathbb{Y}) \rightarrow \mathscr{W}^{1, p}\left(\mathbb{X}, \mathbb{Y}^{\prime}\right)$ and its inverse $F_{\sharp}^{-1}: \mathscr{W}^{1, p}\left(\mathbb{X}, \mathbb{Y}^{\prime}\right) \rightarrow$ $\mathscr{W}^{1, p}(\mathbb{X}, \mathbb{Y})$ are continuous for all $1<p<\infty$. 
Let us comment on some related results. First consider the Sobolev space $\mathscr{W}^{1, p}(\mathbb{X}, \mathbb{R})$ of real-valued functions. If $F: \mathbb{R} \rightarrow \mathbb{R}$ is Lipschitz then the composition $F \circ u$ with $u \in \mathscr{W}^{1, p}(\mathbb{X}, \mathbb{R})$ results in a Sobolev function whose gradient is computed by the rule

$$
\nabla(F \circ u)= \begin{cases}F^{\prime}(u(x)) \nabla u & \text { if } F \text { is differentiable at } u(x), \\ 0 & \text { otherwise. }\end{cases}
$$

The point is that $F$ is differentiable everywhere except for a set $E \subset \mathbb{R}$ of zero linear measure. The preimage $u^{-1}(E) \subset \mathbb{X}$ may have a positive Lebesgue measure where $F^{\prime}$ may not be defined. What comes to the rescue is that $\nabla u$ vanishes on $u^{-1}(E)$ anyway. We refer the reader to a paper by Marcus and Mizel [54] in which they show that in fact the induced operator

$$
F_{\sharp}: \mathscr{W}^{1, p}(\mathbb{X}, \mathbb{R}) \rightarrow \mathscr{W}^{1, p}(\mathbb{X}, \mathbb{R}), \quad F_{\sharp}(u)=F \circ u,
$$

is continuous. Now, suppose that a Lipschitz map $F: \mathbb{Y} \stackrel{\text { onto }}{\longrightarrow} \mathbb{Y}^{\prime}$ is $\mathscr{C}^{1}$-smooth everywhere except for a set $E \subset \mathbb{Y}$ of isolated points. Then the induced composition map

$$
F_{\sharp}: \mathscr{W}^{1, p}(\mathbb{X}, \mathbb{Y}) \rightarrow \mathscr{W}^{1, p}\left(\mathbb{X}, \mathbb{Y}^{\prime}\right), \quad F_{\sharp}(g)=F \circ g,
$$

with $g \in \mathscr{W}^{1, p}(\mathbb{X}, \mathbb{Y})$ is still well defined. The differential is given by

$$
D(F \circ g)= \begin{cases}D F(g(x)) D g(x) & \text { whenever } g(x) \notin E, \\ 0 & \text { otherwise }\end{cases}
$$

The preimage $g^{-1}(E)$ may have positive measure, but $D g(x)$ still vanishes on this set. Because of this it is not difficult to see that the operator (3.4) is continuous.

Proof of Proposition 3.1. We first approximate $\mathbb{Y}$ by polygonal domains. Let $\Upsilon_{v}$ be one of the boundaries of $\mathbb{Y}$. Consider a partition of $\Upsilon_{v}$ into closed Jordan arcs $\widehat{A_{1} A_{2}}, \widehat{A_{2} A_{3}}, \ldots, \widehat{A_{n-1} A_{n}}, \widehat{A_{n} A_{n+1}}, A_{n+1}=A_{1}$, defined by a sequence of consecutive points $A_{1}, \ldots, A_{n} \in \Upsilon_{v}$. Associated with such a partition is a polygonal chain with vertices at $A_{1}, \ldots, A_{n}$, that is, a piecewise linear curve $P_{v}$ that consists of the line segments $\overline{A_{1} A_{2}}, \overline{A_{2} A_{3}}, \ldots, \overline{A_{n-1} A_{n}}, \overline{A_{n} A_{1}}$ connecting the endpoints of the arcs. Although the union $\Upsilon_{v}=\bigcup_{i=1}^{n} \widehat{A_{i} A_{i+1}}$ is a closed Jordan curve, the polygonal chain may have points of self-intersection. However, since $\Upsilon_{v}$ is Lipschitz regular, there is a small $\epsilon>0$ such that if

$$
\operatorname{dist}\left(A_{1}, A_{2}\right) \leq \epsilon, \ldots, \operatorname{dist}\left(A_{n}, A_{1}\right) \leq \epsilon
$$

then only the consecutive segments in the sequence $\overline{A_{1} A_{2}}, \overline{A_{2} A_{3}}, \ldots, \overline{A_{n-1} A_{n}}, \overline{A_{n} A_{1}}$ may intersect, of course, at their common endpoint. Under the condition (3.5) we obtain the boundary of a simply connected domain, call it polygonal. By taking $\epsilon>0$ sufficiently small we may, and do, ensure that each arc $\widehat{A_{1} A_{2}}, \ldots, \widehat{A_{n} A_{1}}$ represents (upon a rotation) the graph of a Lipschitz function; points slightly above the graph lie in $\mathbb{Y}$ and points 
slightly below the graph lie in $\mathbb{R}^{2} \backslash \overline{\mathbb{Y}}$. Fix one of the arcs $\widehat{A_{i} A_{i+1}}$, say the graph of a Lipschitz function $y=f(x), a \leq x \leq b$. Here, for some small positive $\delta$, we have

$$
\begin{aligned}
& \Omega^{+}=\{(x, y): a \leq x \leq b, f(x) \leq y \leq f(x)+\delta\} \subset \overline{\mathbb{Y}}, \\
& \Omega^{-}=\{(x, y): a \leq x \leq b, f(x)-\delta \leq y \leq f(x)\} \subset \mathbb{R}^{2} \backslash \mathbb{Y} .
\end{aligned}
$$

With such a $\delta$ fixed we may, if needed, further partition the arc $\widehat{A_{i} A_{i+1}}$ into a finite number of consecutive subarcs

$$
\widehat{A_{j} A_{j+1}}=\widehat{A_{j} B_{1}} \cup \widehat{B_{1} B_{2}} \cup \cdots \cup \widehat{B_{k} A_{j+1}}
$$

so that each straight line segment $\overline{A_{j} B_{1}}, \overline{B_{1} B_{2}}, \ldots, \overline{B_{k} A_{j+1}}$ lies strictly below the graph of the function $y=f(x)+\delta$ and strictly above the graph of the function $y=f(x)-\delta$. However, to simplify the writing we assume that the arc $\widehat{A_{j} A_{j+1}}$ already enjoys this property. Thus we have the region

$$
\Omega=\Omega^{+} \cup \Omega^{-}=\{(x, y): a \leq x \leq b, f(x)-\delta \leq y \leq f(x)+\delta\}
$$

and two cross-cuts with endpoints $A_{j}=(a, f(a))$ and $A_{j+1}=(b, f(b))$. One cross-cut is the graph of $y=f(x), a \leq x \leq b$, and the other is the straight line segment $\overline{A_{j} A_{j+1}}$, which we shall view as the graph over $[a, b]$ of the linear function $\varphi=\varphi(x)$,

$$
\varphi(x)=\frac{(x-a) f(b)+(b-x) f(a)}{b-a} \quad \text { for all } x \in \mathbb{R} .
$$

Note that $f(x)-\delta<\varphi(x)<f(x)+\delta$ for $a \leq x \leq b$. It will be convenient to extend $f$ as a Lipschitz function on the entire real line simply by setting $f(x)=\varphi(x)$ outside $[a, b]$.

We are now in a position to define a bi-Lipschitz mapping $\Phi: \mathbb{R}^{2} \rightarrow \mathbb{R}^{2}$ associated with the arc $\widehat{A_{j} A_{j+1}} \subset \partial \mathbb{Y}$,

$$
\Phi(x, y)=\left(x, y^{\prime}\right) \quad \text { for }-\infty<x, y<\infty,
$$

where

$$
\begin{aligned}
y^{\prime} & =y+\frac{\varphi(x)-f(x)}{2 \delta}(|y-f(x)-\delta|-2|y-f(x)|+|y-f(x)+\delta|) \\
& = \begin{cases}y, & y \geq f(x)+\delta, \\
\frac{f(x)+\delta-\varphi(x)}{\delta} y+\frac{f(x)+\delta}{\delta}[\varphi(x)-f(x)], & f(x) \leq y \leq f(x)+\delta, \\
\frac{\varphi(x)+\delta-f(x)}{\delta} y+\frac{f(x)-\delta}{\delta}[f(x)-\varphi(x)], & f(x)-\delta \leq y \leq f(x), \\
y, & y \leq f(x)-\delta .\end{cases}
\end{aligned}
$$

We see from this formula that if $x$ is fixed the function $y^{\prime}=y^{\prime}(x, y)$ is piecewise linear and strictly increasing in $y$. Thus $\Phi$ is bi-Lipschitz. Moreover, $\Phi(x, y)=(x, y)$ for all $(x, y) \in \mathbb{R}^{2} \backslash \Omega$, which is immediate when $y \geq f(x)+\delta$ and $y \leq f(x)-\delta$. On the other hand, if $x \notin(a, b)$ then $\varphi(x)=f(x)$, and so $y^{\prime}=y$ from the first formula. Also 
note that $\Phi$ takes the arc $\widehat{A_{j} A_{j+1}}$ into the line segment connecting $A_{j}$ and $A_{j+1}$. Indeed, for $y=f(x)$ we have $\Phi(x, f(x))=(x, \varphi(x))$. An important feature of this particu$\operatorname{lar}$ map $\Phi: \mathbb{R}^{2} \rightarrow \mathbb{R}^{2}$ is that it induces a continuous operator $\Phi_{\sharp}: \mathscr{W}^{1, p}\left(\mathbb{X}, \mathbb{R}^{2}\right) \stackrel{\text { onto }}{\longrightarrow}$ $\mathscr{W}^{1, p}\left(\mathbb{X}, \mathbb{R}^{2}\right)$. To see this consider a sequence $h_{k}(z)=u_{k}+i v_{k}(z)$ converging strongly in $\mathscr{W}^{1, p}\left(\mathbb{X}, \mathbb{R}^{2}\right)$ to $h(x)=u(x)+i v(x)$. We have

$$
\begin{aligned}
& \Phi\left(u_{k}, v_{k}\right) \\
& =\left(u_{k}, v_{k}+\frac{\varphi\left(u_{k}\right)-f\left(u_{k}\right)}{2 \delta}\left(\left|v_{k}-f\left(u_{k}\right)-\delta\right|-2\left|v_{k}-f\left(u_{k}\right)\right|+\left|v_{k}-f\left(u_{k}\right)+\delta\right|\right)\right) \\
& \rightarrow\left(u, v+\frac{\varphi(u)-f(u)}{2 \delta}(|v-f(u)-\delta|-2|v-f(u)|+|v-f(u)+\delta|)\right) \\
& =\Phi(u, v), \quad \text { by (3.3). }
\end{aligned}
$$

The final step in the construction of the map $F: \mathbb{R}^{2} \rightarrow \mathbb{R}^{2}$ consists in dividing the entire boundary $\partial \mathbb{Y}$ into a finite number of sufficiently small arcs $\Gamma_{1}, \ldots, \Gamma_{N} \subset \partial \mathbb{Y}$. To each arc $\Gamma_{i}$ there corresponds a bi-Lipschitz map $\Phi^{i}: \mathbb{R}^{2} \stackrel{\text { onto }}{\longrightarrow} \mathbb{R}^{2}, i=1, \ldots, N$, which takes $\Gamma_{i}$ into a line segment connecting the endpoints of $\Gamma_{i}$. Note that $\Phi^{i}$ is the identity map on all remaining arcs. Therefore, the composition

$$
F:=\Phi^{1} \circ \cdots \circ \Phi^{N}: \mathbb{R}^{2} \stackrel{\text { onto }}{\longrightarrow} \mathbb{R}^{2}
$$

is a bi-Lipschitz map which takes $\mathbb{Y}$ onto a polygonal domain, denote it by $\mathbb{Y}_{\diamond}$. The induced operator

$$
F_{\sharp}=\Phi_{\sharp}^{1} \circ \cdots \circ \Phi_{\sharp}^{N}: \mathscr{W}^{1, p}\left(\mathbb{X}, \mathbb{R}^{2}\right) \rightarrow \mathscr{W}^{1, p}\left(\mathbb{X}, \mathbb{R}^{2}\right)
$$

is continuous. The same applies to the inverse map $F^{-1}$; it induces a continuous operator $F_{\sharp}^{-1}$ as well. Lastly, we compose $F$ with a bi-Lipschitz map $G: \mathbb{R}^{2} \rightarrow \mathbb{R}^{2}$ which is a $\mathscr{C}^{\infty}$-diffeomorphism outside the corners of $\mathbb{Y}_{\diamond}$ and takes this polygon into a circular domain $\mathbb{Y}^{\prime}$. Recall that the operator of composition with $G$ is continuous, by (3.4). All the above compositions result in a desired map $G \circ F: \mathbb{R}^{2} \rightarrow \mathbb{R}^{2}$ which takes $\mathbb{Y}$ into a circular domain $\mathbb{Y}^{\prime}$.

Complete justification of the reduction to Schottky domains goes as follows. Suppose we are given a sequence $\left\{h_{j}\right\} \subset \mathscr{H}_{p}(\mathbb{X}, \mathbb{Y})$ weakly converging to $h \in \widetilde{\mathscr{H}}_{p}(\mathbb{X}, \mathbb{Y})$, thus uniformly as well (see §2.2). Consider the sequence $\left\{H_{j}\right\} \subset \mathscr{H}_{p}\left(\mathbb{X}^{\prime}, \mathbb{Y}^{\prime}\right)$ of homeomorphisms between Schottky domains, defined by $H_{j}=\left(F_{\sharp} \circ T_{b}\right) h_{j}$. This sequence converges weakly to $H=\left(F_{\sharp} \circ T_{b}\right) h \in \mathscr{H}_{p}\left(\mathbb{X}^{\prime}, \mathbb{Y}^{\prime}\right)$, because it is bounded in $\mathscr{W}^{1, p}\left(\mathbb{X}^{\prime}, \mathbb{R}^{2}\right)$ and its uniform limit equals $H$. Once Theorem 1.2 for Schottky domains is in hand, no further work is required. Indeed, we have homeomorphisms $H_{j}^{*} \in \mathscr{H}_{p}\left(\mathbb{X}^{\prime}, \mathbb{Y}^{\prime}\right)$ converging strongly to $H$. Then the sequence of homeomorphisms $h_{j}^{*}:=\left(T_{b}^{-1} \circ F_{\sharp}^{-1}\right) H_{j}^{*} \in$ $\mathscr{H}_{p}(\mathbb{X}, \mathbb{Y})$ serves for Theorem 1.2 in its general setting.

From now on, the components $\mathfrak{X}_{1}, \ldots, \mathfrak{X}_{\ell} \subset \partial \mathbb{X}$ and $\Upsilon_{1}, \ldots, \Upsilon_{\ell} \subset \partial \mathbb{Y}$ are circles. These circles are so numbered that the mappings $h_{j} \in \mathscr{H}_{p}(\mathbb{X}, \mathbb{Y})$ and their weak limit 
$h \in \widehat{\mathscr{H}}_{p}(\mathbb{X}, \mathbb{Y})$ take $\mathfrak{X}_{\nu}$ onto $\Upsilon_{\nu}$ :

$$
h_{j}\left(\mathfrak{X}_{v}\right)=\Upsilon_{\nu} \quad \text { and } \quad h\left(\mathfrak{X}_{v}\right)=\Upsilon_{\nu}, \quad v=1, \ldots, \ell,
$$

for sufficiently large $j$. To simplify the writing we allow $j=1,2, \ldots$.

Remark 3.2. Later, there will be a reason to assume, so we do it now, that the centers of the outer boundary circles of $\mathbb{X}$ and $\mathbb{Y}$ lie outside those domains. For this we need $\ell \geq 2$. The case $\ell=1$ will be treated differently.

\subsection{Extension to the Riemann sphere}

Here, with the usual convention concerning the point $\infty$, the one-point compactification $\widehat{\mathbb{C}}=\mathbb{C} \cup\{\infty\}$, equipped with the chordal metric, will be identified with $\mathbb{S}^{2}$. Let $h: \overline{\mathbb{X}} \stackrel{\text { onto }}{\longrightarrow} \overline{\mathbb{Y}}$ be an arbitrary continuous monotone map satisfying the boundary correspondence (3.6). There is a natural way to extend it to a monotone map $\widehat{h}: \widehat{\mathbb{C}} \stackrel{\text { onto }}{\longrightarrow} \widehat{\mathbb{C}}$.

Each boundary circle $\mathfrak{X}_{i}=\left\{z:\left|z-z_{i}\right|=r_{i}\right\}$ provides a reflection

$$
\varphi_{i}: \widehat{\mathbb{C}} \rightarrow \widehat{\mathbb{C}}, \quad \varphi_{i}(z)=\frac{z-z_{i}}{\left|z-z_{i}\right|^{2}} r_{i}^{2}+z_{i}, \quad i=1, \ldots, \ell,
$$

and a domain $\mathbb{X}_{i}=\varphi_{i}(\mathbb{X})$ which is attached to $\mathbb{X}$ on the other side of the circle. In this way $\overline{\mathbb{X}}$ is furnished with its neighborhood

$$
\mathbb{X}_{+}=\overline{\mathbb{X}} \cup \mathbb{X}_{1} \cup \cdots \cup \mathbb{X}_{\ell}
$$

This is a circular domain of connectivity $\ell(\ell-1)$ whose boundary components are

$$
\mathfrak{X}_{i}^{j}=\varphi_{i}\left(\mathfrak{X}_{j}\right), \quad i \neq j, \quad \partial \mathbb{X}_{+}=\bigcup_{i \neq j} \mathfrak{X}_{i}^{j}
$$

The same construction works for the circles $\Upsilon_{i} \subset \partial \mathbb{Y}$ in the target domain; we have the reflections $\psi_{i}: \widehat{\mathbb{C}} \rightarrow \widehat{\mathbb{C}}$, the domains $\mathbb{Y}_{i}=\psi_{i}(\mathbb{Y}), i=1, \ldots, \ell$, and a neighborhood of $\overline{\mathbb{Y}}$ :

$$
\mathbb{Y}_{+}=\overline{\mathbb{Y}} \cup \mathbb{Y}_{1} \cup \cdots \cup \mathbb{Y}_{\ell}, \quad \partial \mathbb{Y}_{+}=\bigcup_{i \neq j} \Upsilon_{i}^{j}
$$

Now we define the extension $\widehat{h}: \overline{\mathbb{X}}_{+} \stackrel{\text { onto }}{\longrightarrow} \overline{\mathbb{Y}}_{+}$of $h: \overline{\mathbb{X}} \rightarrow \bar{Y}$ by the rule

$$
\widehat{h}=\psi_{i} \circ h \circ \varphi_{i}: \overline{\mathbb{X}}_{i} \rightarrow \overline{\mathbb{Y}}_{i} \quad \text { for } i=1, \ldots, \ell .
$$

Since $h: \overline{\mathbb{X}} \rightarrow \overline{\mathbb{Y}}$ is monotone, so is the extended mapping $\widehat{h}: \overline{\mathbb{X}}_{+} \stackrel{\text { onto }}{\longrightarrow} \overline{\mathbb{Y}}_{+}$. Furthermore, whenever $h: \mathbb{X} \stackrel{\text { onto }}{\longrightarrow} \mathbb{Y}$ is a homeomorphism, the extended mappings $\widehat{h}: \mathbb{X}_{i} \stackrel{\text { onto }}{\longrightarrow} \mathbb{Y}_{i}$, $i=1, \ldots, \ell$, are homeomorphisms. The boundary mappings $h: \mathfrak{X}_{i} \stackrel{\text { onto }}{\longrightarrow} \Upsilon_{i}$ are also monotone; this implies that $\widehat{h}: \mathfrak{X}_{i}^{j} \stackrel{\text { onto }}{\longrightarrow} \Upsilon_{i}^{j}, i \neq j$, is monotone as well. 
3.3.1. A supplementary extension $\widehat{h}: \overline{\mathbb{C}} \stackrel{\text { onto }}{\longrightarrow} \overline{\mathbb{C}}$. Proceeding in this direction, we further extend $\widehat{h}: \overline{\mathbb{X}}_{+} \stackrel{\text { onto }}{\longrightarrow} \overline{\mathbb{Y}}_{+}$to the entire Riemann sphere $\widehat{\mathbb{C}}$, obtaining a map again denoted by $\widehat{h}: \widehat{\mathbb{C}} \stackrel{\text { onto }}{\longrightarrow} \widehat{\mathbb{C}}$. We need this additional extension only to provide easy references to the theory of monotone mappings on $\mathbb{S}^{2}$. There will be no need for any regularity of $\widehat{h}$ outside $\mathbb{X}_{+}$.

Let $\mathbb{X}_{i}^{j}$ and $\mathbb{Y}_{i}^{j}, i \neq j$, denote the components of $\widehat{\mathbb{C}} \backslash \mathbb{X}^{+}$and $\widehat{\mathbb{C}} \backslash \mathbb{Y}^{+}$at the opposite sides of the boundary circles $\mathfrak{X}_{i}^{j}$ and $\Upsilon_{i}^{j}$, respectively. We then extend the boundary mappings $\widehat{h}: \mathfrak{X}_{i}^{j} \stackrel{\text { onto }}{\longrightarrow} \Upsilon_{i}^{j}, i \neq j$, in a radial fashion to those components, and continue to write $\widehat{h}: \mathbb{X}_{i}^{j} \stackrel{\text { onto }}{\longrightarrow} \mathbb{Y}_{i}^{j}, i \neq j$.

Of course, continuity and monotonicity of $\widehat{h}: \widehat{\mathbb{C}} \rightarrow \widehat{\mathbb{C}}$ are understood with respect to the chordal metric. Observe that uniform convergence of continuous mappings $h_{j}: \overline{\mathbb{X}} \stackrel{\text { onto }}{\longrightarrow} \overline{\mathbb{Y}}, h_{j}: \mathfrak{X}_{v} \rightarrow \Upsilon_{v}, v=1, \ldots, \ell, j=1,2, \ldots$, to $h: \overline{\mathbb{X}} \stackrel{\text { onto }}{\longrightarrow} \overline{\mathbb{Y}}$ yields uniform convergence of $\widehat{h}_{j}: \widehat{\mathbb{C}} \stackrel{\text { onto }}{\longrightarrow} \widehat{\mathbb{C}}$ to $\widehat{h}: \widehat{\mathbb{C}} \stackrel{\text { onto }}{\longrightarrow} \widehat{\mathbb{C}}$.

A theorem of Kuratowski and Lacher [48, 49] asserts that:

Theorem 3.3. Let $\mathbf{X}$ and $\mathbf{Y}$ be compact Hausdorff spaces, $\mathbf{Y}$ being locally connected. Suppose we are given a sequence of monotone mappings $f_{k}: \mathbf{X} \stackrel{\text { onto }}{\longrightarrow} \mathbf{Y}$ (e.g. homeomorphisms) converging uniformly to a mapping $f: \mathbf{X} \rightarrow \mathbf{Y}$. Then $f: \mathbf{X} \stackrel{\text { onto }}{\longrightarrow} \mathbf{Y}$ is monotone.

Throughout this text, we shall freely use, without explicit mention, basic topological properties of monotone mappings of $\mathbb{S}^{2}$. Nevertheless, when clarity requires it, we shall provide an explanation.

Proposition 3.4. Let $f: \widehat{\mathbb{C}} \stackrel{\text { onto }}{\longrightarrow} \widehat{\mathbb{C}}$ be a continuous monotone map such that $f: \mathbb{C} \stackrel{\text { onto }}{\longrightarrow} \mathbb{C}$. If $\Gamma \subset \mathbb{C}$ is a continuum that disconnects $\mathbb{C}$ into two components then so is $f^{-1}(\Gamma)$.

\section{Analytical preliminaries}

At this point we must specify our definition of $p$-harmonic mappings.

\section{1. p-harmonic mappings}

Let $h=u+i v$ be a complex-valued function in the Sobolev space $\mathscr{W}^{1, p}(\mathbb{X}, \mathbb{C})$, $1<p<\infty$. We consider the gradient map $\nabla h=(\nabla u, \nabla v): \mathbb{X} \stackrel{\text { into }}{\longrightarrow} \mathbb{R}^{2} \times \mathbb{R}^{2}$, its modulus $|\nabla h|=\left(|\nabla u|^{p}+|\nabla v|^{p}\right)^{1 / p}$ and the $\mathscr{L}^{p}$-energy

$$
\mathcal{E}_{\mathbb{X}}[h]=\iint_{\mathbb{X}}|\nabla h(x)|^{p} d x=\iint_{\mathbb{X}}\left(|\nabla u(x)|^{p}+|\nabla v(x)|^{p}\right) d x=\|\nabla h\|_{p}^{p} .
$$

The reason for choosing exactly this type of $\mathscr{L}^{p}$-energy is that the associated LagrangeEuler system consists of two scalar $p$-harmonic equations

$$
\operatorname{div}|\nabla u|^{p-2} \nabla u=0 \quad \text { and } \quad \operatorname{div}|\nabla v|^{p-2} \nabla v=0 .
$$


Definition 4.1. A complex-valued function $h=u+i v$ of $\operatorname{Sobolev}$ class $\mathscr{W}_{\mathrm{loc}}^{1, p}(\Omega, \mathbb{C})$, $1<p<\infty$, is called a $p$-harmonic map if it satisfies the uncoupled system of equations (4.2).

We shall take advantage of this setting by considering the complex gradients $\partial u / \partial z$ and $\partial v / \partial z$, which turn out to be quasiregular mappings. Their topological properties will play a vital role in the proof of a $p$-harmonic variant of Hurwitz theorems.

\subsection{Modulus of continuity}

When $p>2$, just the Sobolev inequality gives a uniform bound of the modulus of continuity in terms of $\mathscr{L}^{p}$-energy:

Lemma 4.2. For every Sobolev mapping $h \in \mathscr{W}^{1, p}(\mathbb{X}, \mathbb{C}), p>2$, in a bounded Lipschitz domain $\mathbb{X} \subset \mathbb{R}^{2}$ we have

$$
\left|h\left(x_{1}\right)-h\left(x_{2}\right)\right|^{p} \leq C_{p}(\mathbb{X})\left|x_{1}-x_{2}\right|^{p-2} \iint_{\mathbb{X}}|\nabla h|^{p}, \quad x_{1}, x_{2} \in \overline{\mathbb{X}} .
$$

When $p=2$, monotonicity of Sobolev mappings lets us prove similar estimates in terms of the Dirichlet energy. The multiply connected case, $\ell \geq 2$, is easy to handle via uniform energy bounds in a neighborhood of $\overline{\mathbb{X}}$,

$$
\iint_{\mathbb{X}_{+}}|\nabla \widehat{h}|^{2} \leq C(\mathbb{X}, \mathbb{Y}) \iint_{\mathbb{X}}|\nabla h|^{2}
$$

The neighborhood $\mathbb{X}_{+}=\overline{\mathbb{X}} \cup \mathbb{X}_{1} \cup \cdots \cup \mathbb{X}_{\ell}$ and the extended map $\widehat{h}: \mathbb{X}_{+} \stackrel{\text { onto }}{\longrightarrow} \mathbb{Y}_{+}$are obtained via the reflections $\varphi_{i}: \mathbb{X} \stackrel{\text { onto }}{\longrightarrow} \mathbb{X}_{i}$ and $\psi_{i}: \mathbb{Y} \stackrel{\text { onto }}{\longrightarrow} \mathbb{Y}_{i}$, which are $\mathscr{C}^{\infty}$-smooth diffeomorphisms up to the boundary (recall that the centers of the outer circles lie outside $\mathbb{X}$ and $\mathbb{Y}$, and that is why we need $\ell \geq 2$ ).

We may now use local estimates for monotone mapping $\widehat{h}$ in the Sobolev space $\mathscr{W}^{1,2}\left(\mathbb{X}_{+}, \mathbb{R}^{2}\right)$ (see $[34$, Corollary 7.5 .1$\left.]\right)$ to obtain

Lemma 4.3. For $h \in \mathscr{H}_{2}(\mathbb{X}, \mathbb{Y})$ and $\ell \geq 2$,

$$
\left|h\left(x_{1}\right)-h\left(x_{2}\right)\right|^{2} \leq \frac{C_{2}(\mathbb{X}, \mathbb{Y})}{\log \left(1+\frac{\operatorname{diam} \mathbb{X}}{\left|x_{1}-x_{2}\right|}\right)} \iint_{\mathbb{X}}|\nabla h|^{2}, \quad x_{1}, x_{2} \in \overline{\mathbb{X}}
$$

Remark 4.4. Inequality (4.5), and hence Theorem 1.2, fail without the additional requirement when $\ell=1$ and $p=2$. Consider the Möbius transformations $h_{k}: \mathbb{D} \stackrel{\text { onto }}{\longrightarrow} \mathbb{D}$ (normalized at two boundary points)

$$
h_{k}(z)=\frac{z+a_{k}}{1+a_{k} z}, \quad h_{k}(1)=1, \quad h_{k}(-1)=-1, \quad \mathcal{E}_{\mathbb{D}}\left[h_{k}\right]=2 \pi,
$$

where $0<a_{k}<1$. As $a_{k}$ approaches 1 the mappings converge to $h(z) \equiv 1, c$-uniformly and weakly in $\mathscr{W}^{1,2}(\mathbb{D})$. Obviously, the constant map cannot be obtained as a strong $\mathscr{W}^{1,2}(\mathbb{D})$-limit of homeomorphisms of $\mathbb{D}$ onto itself. We are losing equicontinuity of the boundary mappings $h_{k}: \partial \mathbb{D} \stackrel{\text { onto }}{\longrightarrow} \partial \mathbb{D}$. 
Lemma 4.5. Inequality (4.5) still holds for $\ell=1$ if the mapping $h \in \mathscr{H}_{2}(\mathbb{X}, \mathbb{Y})$ is properly normalized, say being fixed at a given point in $\mathbb{X}$ or at three preassigned points in $\partial \mathbb{X}$; the constant $C_{2}(\mathbb{X}, \mathbb{Y})$ depends on the normalization.

Proof. We argue for (4.5) in each of the two cases separately.

4.2.1. A normalization at the interior point. Consider homeomorphisms $h \in \mathscr{H}_{2}(\mathbb{X}, \mathbb{Y})$ between simply connected Lipschitz domains, which are fixed at a given point inside $\mathbb{X}$,

$$
h\left(x_{\circ}\right)=y_{\circ}, \quad \text { where } x_{\circ} \in \mathbb{X} \text { and } y_{\circ} \in \mathbb{Y} .
$$

With the aid of a bi-Lipschitz transformation (Section 3.2) we are reduced to a selfhomeomorphism of the unit disk, still denoted by $h: \mathbb{D} \stackrel{\text { onto }}{\longrightarrow} \mathbb{D}$, with $h(0)=0$. From [39, Theorem 8.1] we deduce the inequality

$$
\operatorname{dist}^{2}(h(z), \partial \mathbb{D}) \leq \frac{C}{\log \left(e+\frac{2}{\operatorname{dist}(z, \partial \mathbb{D})}\right)} \iint_{\mathbb{D}}|\nabla h|^{2}
$$

where $C \geq 1$ is an absolute constant.

Case 1. Suppose that

$$
\mathcal{E}:=\iint_{\mathbb{D}}|\nabla h|^{2} \geq \frac{1}{4 C} \log \left(1+\frac{2}{\left|x_{1}-x_{2}\right|}\right) .
$$

Inequality (4.5) holds with any constant $C_{2}(\mathbb{X}, \mathbb{Y}) \geq 4 C \operatorname{diam}^{2} \mathbb{Y}=16 C$.

Case 2. Suppose that

$$
\mathcal{E}=\iint_{\mathbb{D}}|\nabla h|^{2}<\frac{1}{4 C} \log \left(1+\frac{2}{\left|x_{1}-x_{2}\right|}\right), \quad \text { obviously } \quad \mathcal{E} \geq 2|\mathbb{D}|=2 \pi .
$$

Therefore $x_{1}, x_{2} \in B(a, r) \subset B(a, 2 r)$, where $B \subset 2 B$ are concentric disks centered at $a=\frac{1}{2}\left(x_{1}+x_{2}\right) \in \mathbb{D}$ and $r=\frac{1}{2}\left|x_{1}-x_{2}\right|<\left(e^{8 \pi C}-1\right)^{-1}<1 / 2$.

Now introduce a continuous monotone extension $\widehat{h}: \widehat{\mathbb{C}} \rightarrow \widehat{\mathbb{C}}$,

$$
\widehat{h}(z)=[\overline{h(1 / \bar{z})}]^{-1} \quad \text { for }|z| \geq 1 .
$$

For $z \in B(a, 2 r) \backslash \mathbb{D}$, we have $1<|z|<1+2 r<2$. It then follows from (4.7) that

$$
[1-|h(1 / \bar{z})|]^{2} \leq \frac{C \mathcal{E}}{\log \left(e+\frac{2|z|}{|z|-1}\right)}<\frac{\log (1+1 / r)}{4 \log \left(e+\frac{1+2 r}{r}\right)}<\frac{1}{4} .
$$

Hence $|h(1 / \bar{z})|>1 / 2$ and $|\nabla \widehat{h}(z)| \leq|\nabla h(1 / \bar{z})| \cdot|h(1 / \bar{z})|^{-2} \leq 4|\nabla h(1 / \bar{z})|$. The change of variable $z=1 / \xi$ gives

$$
\iint_{B(a, 2 r) \backslash \mathbb{D}}|\nabla \widehat{h}|^{2} \leq 256 \iint_{\mathbb{D}}|\nabla h|^{2}, \quad \text { hence } \iint_{B(a, 2 r)}|\nabla \widehat{h}|^{2} \leq 512 \iint_{\mathbb{D}}|\nabla h|^{2} .
$$

Now (4.5) is immediate from [34, Corollary 7.5.1]. 
4.2.2. A normalization at three boundary points. Let $h \in \mathscr{H}_{2}(\mathbb{X}, \mathbb{Y})$ be normalized by three conditions: $h\left(x_{1}\right)=y_{1}, h\left(x_{2}\right)=y_{2}$ and $h\left(x_{3}\right)=y_{3}$, where we are given distinct points $x_{1}, x_{2}, x_{3} \in \partial \mathbb{X}$ and $y_{1}, y_{2}, y_{3} \in \partial \mathbb{Y}$. We assume that these points are positively oriented along $\partial \mathbb{X}$ and $\partial \mathbb{Y}$, respectively. First we reduce the domains $\mathbb{X}$ and $\mathbb{Y}$ (via biLipschitz transformations) to equilateral triangles in which $\left(x_{1}, x_{2}, x_{3}\right)$ and $\left(y_{1}, y_{2}, y_{3}\right)$ are their vertices. We aim to extend $h$ to a neighborhood $\mathbb{X}_{+} \supset \overline{\mathbb{X}}$. To define such a neighborhood we reflect the triangle $\mathbb{X}$ across its edges, which results in three adjacent triangles. We then reflect the adjacent triangles across their edges, and so forth. Eventually we arrive at 12 equilateral triangles surrounding $\mathbb{X}$, which together with $\overline{\mathbb{X}}$ form a neighborhood denoted by $\mathbb{X}_{+}$. The same reflection procedure is made for $\mathbb{Y}$. We then extend $h: \mathbb{X} \stackrel{\text { onto }}{\longrightarrow} \mathbb{Y}$ in accordance with the above reflections and define continuous monotone extensions $\widehat{h}_{k}: \mathbb{X}_{+} \stackrel{\text { onto }}{\longrightarrow} \mathbb{Y}_{+}$. The way the extensions are made secures a uniform energy bound

$$
\iint_{\mathbb{X}_{+}}|\nabla \widehat{h}|^{2}=13 \iint_{\mathbb{X}}|\nabla h|^{2} \leq M .
$$

By the same arguments as before we conclude with inequality (4.5).

Remark 4.6. In view of (4.3) and (4.5) a sequence $\left\{h_{j}\right\} \subset \mathscr{H}_{p}(\mathbb{X}, \mathbb{Y})$ that is converging weakly to $h$ actually converges uniformly on $\overline{\mathbb{X}}$. Furthermore, $h_{j}: \overline{\mathbb{X}} \stackrel{\text { onto }}{\longrightarrow} \overline{\mathbb{Y}}$ and their boundary mappings $h_{j}: \partial \mathbb{X} \stackrel{\text { onto }}{\longrightarrow} \partial \mathbb{Y}$ are monotone as well. This also applies to the weak limit $h: \overline{\mathbb{X}} \stackrel{\text { onto }}{\longrightarrow} \overline{\mathbb{Y}}$ and its boundary map $h: \partial \mathbb{X} \stackrel{\text { onto }}{\longrightarrow} \partial \mathbb{Y}$. Inequalities (4.3) and (4.5) remain valid for $h \in \widehat{\mathscr{H}}_{p}(\mathbb{X}, \mathbb{Y})$.

4.2.3. Royden $p$-algebra. For notational simplicity we introduce the space $\mathscr{R}^{p}(\mathbb{X})$ which consists of continuous functions on $\overline{\mathbb{X}}$ having finite $p$-energy. The norm is given by

$$
\|h\|_{\mathscr{R} p(\mathbb{X})}=\sup _{x \in \mathbb{X}}|h(x)|+\left(\iint_{\mathbb{X}}|\nabla h(x)|^{p} d x\right)^{1 / p}<\infty .
$$

$\mathscr{R}_{0}^{p}(\mathbb{X})$ is the completion of $\mathscr{C}_{0}^{\infty}(\mathbb{X})$ in this norm; it consists of functions vanishing on $\partial \mathbb{X}$.

Remark 4.7. The proofs above (except for simply connected domains with normalization at the inner point) show that a mapping $h \in \widehat{\mathscr{H}}_{p}(\mathbb{X}, \mathbb{Y})$ admits an extension to a continuous monotone map $\widehat{h}: \mathcal{O} \rightarrow \mathbb{R}^{2}$ defined in a neighborhood $\mathcal{O} \supset \overline{\mathbb{X}}$ which is independent of $h$. This extension retains control on the energy; namely, $\mathcal{E}_{\mathcal{O}}[\widehat{h}] \leq C_{p}(\mathbb{X}, \mathbb{Y}) \mathcal{E}_{\mathbb{X}}[h]$. Concerning the exceptional case, a slight change in the proof actually gives an extension $\widehat{h}: \mathcal{O} \rightarrow \mathbb{R}^{2}$, due to inequality (4.7), but the neighborhood $\mathcal{O}$ depends on the energy of $h$.

In summary, regardless of the case, if we restrict ourselves to mappings in $\widehat{\mathscr{H}}_{p}(\mathbb{X}, \mathbb{Y})$ whose energy is bounded by a given constant, say $\mathcal{E}_{\mathbb{X}}[h] \leq M$, then we still have an extension $\widehat{h}: \mathcal{O} \rightarrow \mathbb{R}^{2}$ to a neighborhood depending only on $M$. The extended map has nonnegative Jacobian and its energy is controlled by $M$. This observation is the key to the following lemma. 


\subsection{Weak- $\mathscr{L}^{1}$ convergence of Jacobians}

Lemma 4.8. If a sequence of mappings $f_{k} \in \widehat{\mathscr{H}}_{p}(\mathbb{X}, \mathbb{Y}), p \geq 2$, converges to $f$ weakly in $\mathscr{W}^{1, p}\left(\mathbb{X}, \mathbb{R}^{2}\right)$ then the Jacobian determinants $J_{f_{k}}=J\left(x, f_{k}\right)$ converge to $J_{f}=J(x, f)$ weakly in $\mathscr{L}^{1}(\mathbb{X})$, meaning that

$$
\iint_{\mathbb{X}} \varphi(x) J\left(x, f_{k}\right) d x \rightarrow \iint_{\mathbb{X}} \varphi(x) J(x, f) d x \quad \text { for every } \varphi \in \mathscr{L}^{\infty}(\mathbb{X}) .
$$

Proof. It has long been known that (4.8) holds for $\varphi \in \mathscr{C}_{0}^{\infty}(\mathbb{X})$. Now, if $p>2$, the sequence $\left\{J\left(x, f_{k}\right)\right\}$ is bounded in $\mathscr{L}^{p / 2}(\mathbb{X})$, and so (4.8) is true for all test functions in the dual space $\mathscr{L}^{p /(p-2)}(\mathbb{X}) \supset \mathscr{L}^{\infty}(\mathbb{X})$ where $\mathscr{C}_{0}^{\infty}(\mathbb{X})$ is dense.

For $p=2$ the proof is not straightforward, but relies on the well known higher integrability property of nonnegative Jacobians [60], which we state as

$$
\iint_{\mathbb{X}} J\left(x, f_{k}\right) \log \left(e+\frac{J\left(x, f_{k}\right)}{\left.\left\|J_{f_{k}}\right\| \mathscr{L}^{1} \mathbb{X}\right)}\right) d x \leq C(\mathbb{X}, \mathcal{O}) \iint_{\mathcal{O}}\left|\nabla f_{k}(x)\right|^{2} d x .
$$

The interested reader is referred to $[19,35]$ for further results in this direction. Now, by similar arguments, estimate (4.9) yields (4.8) for all test functions $\varphi \in \mathscr{L}^{\infty}(\mathbb{X})$. As a particular case, upon setting $\varphi=\chi_{\Omega}$, we see that the averages of $J\left(x, f_{k}\right)$ over any measurable set $\Omega \subset \mathbb{X}$ converge to the average of $J(x, f)$,

$$
\iint_{\Omega} J\left(x, f_{k}\right) d x \rightarrow \iint_{\Omega} J(x, f) d x .
$$

\subsection{Dirichlet problem}

There are two commonly used settings of the Dirichlet problem. The classical one, with continuous boundary data, combines the Perron method and Wiener's criterion of regular points on the boundary of the domain. In the variational approach, on the other hand, one seeks to minimize the $p$-harmonic energy integral over the class of functions in $u+\mathscr{W}_{0}^{1, p}(\Omega)$, where $u \in \mathscr{W}^{1, p}(\Omega)$ is viewed as the boundary data. Even when these two different settings are well defined, the question whether they represent the same solution involves a delicate analysis of the boundary of the domain. Strangely enough, in the widely developed theory of the Dirichlet problem, explicit statements concerning simply connected domains appear to be rare in the literature. The equivalence of these two settings is vital in our approach.

Theorem 4.9. Let $\Omega \subset \mathbb{R}^{2}$ be a bounded simply connected domain and $u \in \mathscr{C}(\bar{\Omega}) \cap$ $\mathscr{W}^{1, p}(\Omega), 1<p<\infty$. Then there exists a unique function $\tilde{u} \in \mathscr{C}(\bar{\Omega}) \cap \mathscr{W}^{1, p}(\Omega)$ that is $p$-harmonic in $\Omega$ and equal to $u$ on $\partial \Omega$. Furthermore, $\tilde{u} \in u+\mathscr{W}_{\circ}^{1, p}(\Omega)$ and

$$
\mathcal{E}_{p}[\tilde{u}] \leq \mathcal{E}_{p}[u] .
$$

Equality occurs if and only if $\tilde{u} \equiv u$. 
Proof. We shall not give all details of the proof. Nevertheless, it is worth remarking (because it is not obvious) that the variational solution $\tilde{u}$ extends continuously up to the boundary. This is because each boundary point of a planar simply connected domain is a regular point for the $p$-Laplace operator $\Delta_{p}$ [25, p. 418]. This issue has been discussed by various writers; we refer here to the book by J. Heinonen, T. Kilpeläinen and O. Martio [28, 6.16] for the boundary regularity and relevant notion of capacities and, in particular, to [27, Lemma 5.3] (see also [47, Lemma 4.1] and [50, Lemma 2]) for a capacity estimate that applies to simply connected domains.

\subsection{Radó-Kneser-Choquet theorems in p-harmonic setting}

The classical Radó-Kneser-Choquet theorem asserts that a planar harmonic homeomorphism is in fact a $\mathscr{C}^{\infty}$-diffeomorphism [23]. The same holds for $p$-harmonic homeomorphisms (see Alessandrini and Sigalotti [3]).

Radó-Kneser-Choquet theory also asserts that a harmonic function $h: \Omega \rightarrow \mathbb{C}$ in a Jordan domain that extends continuously to a homeomorphism of $\partial \Omega$ onto a closed convex curve $\Gamma \subset \mathbb{C}$ is a $\mathscr{C}^{\infty}$-diffeomorphism of $\Omega$ onto the bounded component of $\mathbb{C} \backslash \Gamma$. Numerous works were devoted to the extension of this result to solutions of linear elliptic type systems $[1,2,14,17,52,53]$. It resulted in a generalization to nonlinear PDEs by Alessandrini and Sigalotti [3].

Theorem 4.10. Suppose that $\Omega \subset \mathbb{C}$ is a simply connected Jordan domain, $G$ a bounded convex domain and $h \in \mathscr{C}(\bar{\Omega}) \cap \mathscr{W}_{\mathrm{loc}}^{1, p}(\Omega)$ a p-harmonic map that takes $\partial \Omega$ homeomorphically onto $\partial G$. Then $h: \Omega \stackrel{\text { onto }}{\longrightarrow} G$ is a $\mathscr{C}^{\infty}$-diffeomorphism.

\subsection{Hurwitz's theorem}

Let us begin with the quasiconformal variants of the Hurwitz theorems, well known for holomorphic functions (see [51, II 5.3] and [57, Lemma 3]).

Theorem 4.11. If a sequence of $K$-quasiconformal mappings $\varphi_{n}: \Omega \rightarrow \mathbb{C}$ converges c-uniformly to $\varphi: \Omega \rightarrow \mathbb{C}$, then $\varphi$ is either constant or $K$-quasiconformal.

Theorem 4.12. If a sequence of $K$-quasiregular mappings $\varphi_{n}: \Omega \rightarrow \mathbb{C}$ converges c-uniformly to $\varphi: \Omega \rightarrow \mathbb{C}$, and $\varphi_{n}(z) \neq 0$ for all $z \in \Omega$ and $n=1,2, \ldots$, then $\varphi$ is either identically zero, or $\varphi(z) \neq 0$ for all $z \in \Omega$.

The full analog of Theorem 4.11 fails for solutions of second order PDEs. For example, the uniform limit of the harmonic homeomorphisms $\varphi_{n}(z)=x+(i / n) y$ is neither constant nor a homeomorphism. Nevertheless, the Jacobian determinant of the limit map vanishes identically. Hurwitz's theorem below reflects this example.

Theorem 4.13. If a sequence of p-harmonic orientation preserving homeomorphisms $\varphi_{n}: \Omega \rightarrow \mathbb{C}$ converges $c$-uniformly to $\varphi: \Omega \rightarrow \mathbb{C}$, then either $\varphi$ is a p-harmonic homeomorphism (actually $\mathscr{C}^{\infty}$-diffeomorphism) or $J_{\varphi}(z) \equiv 0$ in $\Omega$. 
Proof. For basic properties of quasiregular mappings in relation to $p$-harmonic functions we refer the reader to [17], and for further reading to [22]. Recall from [17] that the complex gradient

$$
f:=u_{z}=\frac{1}{2}\left(u_{x}-i u_{y}\right), \quad z=x+i y,
$$

of a $p$-harmonic function $u: \Omega \rightarrow \mathbb{R}$ is $K$-quasiregular. Thus $u \in \mathscr{C}_{\text {loc }}^{1, \alpha}(\Omega), \alpha=1 / K$. The complex gradient actually satisfies the quasilinear elliptic equation

$$
\frac{\partial f}{\partial \bar{z}}=\frac{2-p}{2 p}\left[\frac{\bar{f}}{f} \frac{\partial f}{\partial z}+\frac{f}{\bar{f}} \frac{\overline{\partial f}}{\partial z}\right], \quad \text { hence } 1 \leq K \leq p-1 \text {. }
$$

In particular, every sequence $\left\{u^{n}\right\}_{n=1}^{\infty}$ of $p$-harmonic functions converging $c$-uniformly on $\Omega$ actually converges in $\mathscr{C}_{\mathrm{loc}}^{1, \alpha}(\Omega)$. Then, by Theorem 4.12 , if the complex gradients $f^{n}=u_{z}^{n}$ vanish nowhere in $\Omega$ then $f=u_{z}$ is either identically zero or nowhere vanishing. Moreover, $f^{n} \rightarrow f$ uniformly on compact subsets together with first order derivatives. Now, consider the $p$-harmonic homeomorphisms (thus $\mathscr{C}^{\infty}$-diffeomorphisms)

$$
\varphi_{n}:=u^{n}+i v^{n}: \Omega \rightarrow \mathbb{C}
$$

and their limit

$$
\varphi:=u+i v: \Omega \rightarrow \mathbb{C} .
$$

Each $\varphi_{n}$ has positive Jacobian determinant, $J_{\varphi_{n}}(z)=u_{x}^{n} v_{y}^{n}-u_{y}^{n} v_{x}^{n}>0$. Thus the complex gradients (quasiregular mappings)

$$
f^{n}(z):=u_{z}^{n} \quad \text { and } \quad g^{n}(z):=v_{z}^{n}
$$

do not vanish in $\Omega$. In view of Theorem 4.12 the limit functions $f(z)=u_{z}$ and $g(z)=v_{z}$ do not vanish either, unless they are identically zero in which case $J_{\varphi}=u_{x} v_{y}-u_{y} v_{x} \equiv 0$, as claimed. Let us now assume that both $f \neq 0$ and $g \neq 0$ in $\Omega$. We shall show that in this case also $J_{\varphi}(z) \neq 0$ in $\Omega$. Suppose otherwise: $J_{\varphi}\left(z_{\circ}\right)=0$ at some point $z_{\circ} \in \Omega$. Equivalently,

$$
\alpha f\left(z_{\circ}\right)+\beta g\left(z_{\circ}\right)=0 \quad \text { for some real numbers } \alpha, \beta \neq 0 .
$$

Consider the complex functions

$$
F^{n}(z)=\alpha f^{n}(z)+\beta g^{n}(z)
$$

and their limit

$$
F(z)=\alpha f(z)+\beta g(z) \quad\left(\text { recall that } F\left(z_{\circ}\right)=0\right) .
$$

To any real coefficients $\alpha, \beta \neq 0$ there corresponds an elliptic first order system of partial differential equations for $F^{n}$. The derivation of that system goes as follows. 
Using (4.12) and the analogous equation for $g$ we compute

$$
\begin{aligned}
F_{\bar{z}}^{n}= & \frac{2-p}{2 p}\left[\frac{\overline{f^{n}}}{f^{n}} \frac{\partial f^{n}}{\partial z}+\frac{f^{n}}{\overline{f^{n}}} \frac{\overline{\partial f^{n}}}{\partial z}\right] \alpha+\frac{2-p}{2 p}\left[\frac{\overline{g^{n}}}{g^{n}} \frac{\partial g^{n}}{\partial z}+\frac{g^{n}}{\overline{g^{n}}} \frac{\overline{\partial g^{n}}}{\partial z}\right] \beta \\
= & \frac{2-p}{2 p}\left[\frac{\overline{g^{n}}}{g^{n}} \frac{\partial F^{n}}{\partial z}+\frac{g^{n}}{\overline{g^{n}}} \frac{\overline{\partial F^{n}}}{\partial z}\right] \\
& +\frac{2-p}{2 p}\left[\left(\frac{\overline{f^{n}}}{f^{n}}-\frac{\overline{g^{n}}}{g^{n}}\right) \frac{\partial f^{n}}{\partial z}+\left(\frac{f^{n}}{\overline{f^{n}}}-\frac{g^{n}}{\overline{g^{n}}}\right) \frac{\overline{\partial f^{n}}}{\partial z}\right] \alpha .
\end{aligned}
$$

Here we have

$$
\frac{\overline{f^{n}}}{f^{n}}-\frac{\overline{g^{n}}}{g^{n}}=\frac{\left(\alpha f^{n}+\beta g^{n}\right) \overline{f^{n}}-\left(\alpha \overline{f^{n}}+\beta \overline{g^{n}}\right) f^{n}}{\beta f^{n} g^{n}}
$$

and hence

$$
\left|\frac{\overline{f^{n}}}{f^{n}}-\frac{\overline{g^{n}}}{g^{n}}\right| \leq \frac{2\left|F^{n}\right|}{|\beta|\left|g^{n}\right|} .
$$

Thus we have the first order (elliptic) inequality

$$
\left|\frac{\partial F^{n}}{\partial \bar{z}}\right| \leq\left|1-\frac{2}{p}\right|\left|\frac{\partial F^{n}}{\partial z}\right|+\left|1-\frac{2}{p}\right| \frac{2|\alpha|}{|\beta|\left|g^{n}\right|}\left|F^{n}\right| .
$$

This yields a linear equation in $\Omega$,

$$
\frac{\partial F^{n}}{\partial \bar{z}}=\mu^{n}(z) \frac{\partial F^{n}}{\partial z}+A^{n}(z) F^{n},
$$

whose complex measurable coefficients satisfy

$$
\begin{aligned}
& \left|\mu^{n}(z)\right| \leq k=\left|1-\frac{2}{p}\right|<1 \\
& \left|A^{n}(z)\right| \leq 2 k\left|\frac{\alpha}{\beta}\right|\left|\frac{1}{g^{n}}\right|\left|\frac{\partial f^{n}}{\partial z}\right| \in \mathscr{L}_{\mathrm{loc}}^{\infty}(\Omega) .
\end{aligned}
$$

To see this last inclusion we fix a compactly contained subdomain $G \Subset \Omega$. Recall that the functions $g^{n}(z)$ and their $c$-uniform limit $g$ never vanish. This yields a uniform bound from below $\left|g^{n}(z)\right| \geq m$, for $z \in G, m$ being independent of $n=1,2, \ldots$ On the other hand, the continuous functions $\frac{\partial f^{n}}{\partial z}(z)$ converge uniformly on $G$, so we also have the uniform bound $\left|\frac{\partial f^{n}}{\partial z}(z)\right| \leq M$. Therefore we have a pointwise estimate $\left|A^{n}(z)\right| \leq \frac{2 k M|\alpha|}{m|\beta|}$ in $G$, as required.

To deal with equation (4.13) we solve (uniquely) the nonhomogeneous Beltrami equation

$$
\lambda_{\bar{z}}^{n}=\left[\mu^{n}(z) \lambda_{z}^{n}-A^{n}(z)\right] \chi_{G}(z) \text { for a complex function } \lambda^{n} \in \mathscr{W}^{1, s}\left(\mathbb{R}^{2}\right)
$$


where $s>2$. The unknown function $\lambda^{n}(z)$ is represented by the Cauchy transform of a measurable function $\omega^{n} \in \mathscr{L}^{s}\left(\mathbb{R}^{2}\right)$ with $\operatorname{supp} \omega^{n} \subset G \Subset \Omega$ :

$$
\lambda^{n}(z)=\frac{1}{\pi} \iint_{\mathbb{C}} \frac{\omega^{n}(\xi) d \xi}{z-\xi} .
$$

The unknown density function $\omega^{n}$ is found (uniquely) by solving the singular integral equation

$$
\omega^{n}=\mu^{n} \chi_{G} \mathcal{S} \omega^{n}-A^{n} \chi_{G}, \quad \mathcal{S} \omega^{n}=-\frac{1}{\pi} \iint_{\mathbb{C}} \frac{\omega^{n}(\xi)}{(z-\xi)^{2}} d \xi .
$$

Here $\mathcal{S}: \mathscr{L}^{s}(\mathbb{C}) \rightarrow \mathscr{L}^{s}(\mathbb{C})$ is the familiar Beurling-Ahlfors operator. At this stage we appeal to the seminal work of B. Bojarski [16] which provides us with the solution

$$
\omega^{n}=-\left(I-\mu^{n} \chi_{G} \mathcal{S}\right)^{-1} A^{n} \chi_{G} .
$$

Here the operator

$$
I-\mu^{n} \chi_{G} \mathcal{S}: \mathscr{L}^{s}(\mathbb{C}) \rightarrow \mathscr{L}^{s}(\mathbb{C})
$$

is invertible for some $s>2$ (see [7] for the full range $1+k<s<1+1 / k$ ). We see that $\sup _{n}\left\|\omega^{n}\right\| \mathscr{L}^{s}(\mathbb{C})<\infty$ and conclude that the family $\left\{\lambda^{n}\right\}_{n=1}^{\infty}$ is equicontinuous. This follows from basic estimates of the Cauchy transform,

$$
\left|\lambda^{n}\left(z_{1}\right)-\lambda^{n}\left(z_{2}\right)\right| \leq C_{s}\left|z_{1}-z_{2}\right|^{1-2 / s}\left\|\omega^{n}\right\| \mathscr{L}^{s}(\mathbb{C}) .
$$

We may and do assume, by passing to a subsequence if necessary, that $\lambda^{n}(z) \rightrightarrows \lambda(z)$ (uniformly) in $\mathbb{C}$. Therefore,

$$
e^{\lambda^{n}(z)} F^{n}(z) \rightrightarrows e^{\lambda(z)} F(z) \quad \text { uniformly on } G .
$$

Now observe that the nowhere vanishing function $H^{n}(z)=e^{\lambda^{n}} F^{n}$ satisfies the homogeneous Beltrami equation

$$
H_{\bar{z}}^{n}=\mu^{n}(z) H_{z}^{n} \quad \text { in } G .
$$

This is straightforward from (4.13) and (4.14). Thus $H^{n}$ are $K$-quasiregular mappings, $K=\frac{1+k}{1-k}=p-1$. Using Theorem 4.12 we see that the limit function $H(z)=e^{\lambda(z)} F(z)$ does not vanish anywhere in $G$. But $F\left(z_{\circ}\right)=0$, a clear contradiction.

Finally, the inequality $J_{\varphi}(z) \neq 0$ implies that the map $\varphi$ is a local $\mathscr{C}^{1}$-diffeomorphism. Since this map is a $c$-uniform limit of homeomorphisms, it must be a global $\mathscr{C}^{\infty}$-diffeomorphism.

\section{Proof of Theorem 1.2}

Given a mapping $h \in \widehat{\mathscr{H}}_{p}(\mathbb{X}, \mathbb{Y}) \subset \mathscr{R}^{p}(\mathbb{X})$ and $\varepsilon>0$, the goal is to find an injective mapping $h^{*}: \mathbb{X} \stackrel{\text { onto }}{\longrightarrow} \mathbb{Y}$ with $h^{*}-h \in \mathscr{R}_{0}^{p}(\mathbb{X})$ such that

$$
\left\|h^{*}-h\right\|_{\mathscr{R}^{p}(\mathbb{X})} \leq \varepsilon .
$$

It takes several procedures to accomplish this task. 


\subsection{Injectivity in good domains $\mathbb{G}_{v}$}

The construction of $h^{*}$ is by induction on $v=1, \ldots, \ell$. In each step we gain injectivity in a subdomain $\mathbb{G}_{\nu} \subset \mathbb{X}$ that touches the boundary component $\mathfrak{X}_{\nu}$. Let us call it a good domain. Inequality (5.1) is just all those injectivity properties in good domains combined.

Proposition 5.1. To every $f \in \widehat{\mathscr{H}}_{p}(\mathbb{X}, \mathbb{Y})$ and $v=1, \ldots, \ell$, there corresponds a domain $\mathbb{G}=\mathbb{G}_{\nu}:=\left\{x \in \mathbb{X}: f(x) \in \mathbb{Y} \cup \Upsilon_{\nu}\right\}$ such that whenever $\varepsilon>0$ there exists an approximation $f^{*} \in \widehat{\mathscr{H}}_{p}(\mathbb{X}, \mathbb{Y})$ of $f$ such that

$$
\begin{gathered}
\left\|f^{*}-f\right\|_{\mathscr{R}}^{p(\mathbb{X})} \leq \varepsilon / \ell, \quad f^{*}-f \in \mathscr{R}_{0}^{p}(\mathbb{G}) \subset \mathscr{R}_{0}^{p}(\mathbb{X}), \\
f_{\mid \mathbb{G}}^{*}: \mathbb{G} \stackrel{\text { onto }}{\longrightarrow} \mathbb{Y} \quad \text { is injective. }
\end{gathered}
$$

In the above hypotheses, a normalization of $f$ must be included if $\ell=1$ and $p=2$, exactly as in Theorem 1.2 .

Remark 5.2. It involves no loss of generality to assume that the given boundary component $\Upsilon_{\nu} \subset \partial \mathbb{Y}$ is the outer circle; for if not, we reflect $\mathbb{Y}$ across $\Upsilon_{\nu}$. In what follows, we suppress the index $v$ in the notation of the outer circle, writing simply $\Upsilon_{v}=\Upsilon$.

The proof is postponed until Section 5.1. Meanwhile let us demonstrate how the construction of $h^{*} \in \mathscr{H}_{p}(\mathbb{X}, \mathbb{Y})$ goes once Proposition 5.1 is in hand.

5.1.1. Construction of $h^{*}$. We define by induction a chain of mappings $f_{0}, \ldots, f_{\ell} \in$ $\mathscr{\mathscr { H }}_{p}(\mathbb{X}, \mathbb{Y})$ that begins with $f_{0} \equiv h$. The last term in the chain will serve for $h^{*}$. Suppose $f_{v-1} \in \widehat{\mathscr{H}}_{p}(\mathbb{X}, \mathbb{Y})$, for some $1 \leq v \leq \ell$, is given. We appeal to Proposition 5.1 in which $f=f_{v-1}$ and $\mathbb{G}=\mathbb{G}_{v}=\left\{x \in \mathbb{X}: f_{v-1}(x) \in \mathbb{Y} \cup \Upsilon_{\nu}\right\}$, and define $f_{v}=f_{v-1}^{*}$. Take $f_{\ell}$ for $h^{*}$. Obviously $h^{*} \in h+\mathscr{R}_{0}^{p}(\mathbb{X})$ and

$$
\left\|h^{*}-h\right\|_{\mathscr{R} p(\mathbb{X})} \leq \sum_{v=1}^{\ell}\left\|f_{v}-f_{v-1}\right\|_{\mathscr{R} p(\mathbb{X})} \leq \ell \cdot \epsilon / \ell=\epsilon .
$$

Claim 1. $\mathbb{G}_{1} \cup \cdots \cup \mathbb{G}_{\ell}=\mathbb{X}$.

Suppose otherwise: some $x \in \mathbb{X}$ does not lie in $\mathbb{G}_{1} \cup \cdots \cup \mathbb{G}_{\ell}$. Since $x \notin \mathbb{G}_{\ell}$, it follows that $f_{\ell}(x)=f_{\ell-1}(x)$, because $f_{\ell}-f_{\ell-1} \in \mathscr{R}_{0}^{p}\left(\mathbb{G}_{\ell}\right)$. Continuing in this fashion, we see that $f_{\ell-1}(x)=f_{\ell-2}(x)=\cdots=f_{0}(x)=h(x)$. Going backwards we get

$$
\begin{array}{cc}
h(x)=f_{0}(x) \notin \mathbb{Y} \cup \Upsilon_{1}, & \text { because } x \notin \mathbb{G}_{1}, \\
h(x)=f_{1}(x) \notin \mathbb{Y} \cup \Upsilon_{2}, & \text { because } x \notin \mathbb{G}_{2}, \\
\cdots & \\
h(x)=f_{\ell-1}(x) \notin \mathbb{Y} \cup \Upsilon_{\ell}, & \text { because } x \notin \mathbb{G}_{\ell} .
\end{array}
$$

Thus $h(x) \notin \mathbb{Y} \cup \Upsilon_{1} \cup \cdots \cup \Upsilon_{\ell}=\overline{\mathbb{Y}}$, a clear contradiction.

Claim 2. $f_{\ell}: \mathbb{X} \stackrel{\text { onto }}{\longrightarrow} \mathbb{Y}$.

We obviously have $\overline{\mathbb{Y}} \supset f_{\ell}(\mathbb{X}) \supset f_{\ell}\left(\mathbb{G}_{\ell}\right)=\mathbb{Y}$, because $f_{\ell \mid \mathbb{G}_{\ell}}=f_{\ell-1 \mid \mathbb{G}_{\ell}}^{*}=\mathbb{Y}$, by condition (5.3). Suppose that, contrary to the claim, $f_{\ell}(x) \in \partial \mathbb{Y}$ for some $x \in \mathbb{X} \backslash \mathbb{G}_{\ell}$. This 
means that $f_{\ell}(x)=f_{\ell-1}(x) \in \partial \mathbb{Y}$. But $f_{\ell-1}: \mathbb{G}_{\ell-1} \stackrel{\text { onto }}{\longrightarrow} \mathbb{Y}$, so $x \notin \mathbb{G}_{\ell-1}$. Continuing in this way we arrive at $x \notin \mathbb{G}_{\ell-2}, \ldots, x \notin \mathbb{G}_{1}$. This summarizes as $x \notin \mathbb{G}_{1} \cup \cdots \cup \mathbb{G}_{\ell}=\mathbb{X}$, which contradicts Claim 1 .

Claim 3. $f_{\ell}: \mathbb{X} \stackrel{\text { onto }}{\longrightarrow} \mathbb{Y}$ is injective, thus a homeomorphism.

Suppose that, on the contrary, there are $x_{1} \neq x_{2}$ in $\mathbb{X}$ for which $f_{\ell}\left(x_{1}\right)=f_{\ell}\left(x_{2}\right)=$ : $y \in \mathbb{Y}$. Since $f_{\ell}: \mathbb{G}_{\ell} \stackrel{\text { onto }}{\longrightarrow} \mathbb{Y}$ is injective, $x_{1}$ and $x_{2}$ cannot both belong to $\mathbb{G}_{\ell}$. It is also impossible that $x_{1} \in \mathbb{G}_{\ell}$ and $x_{2} \notin \mathbb{G}_{\ell}$, since we would then have $f_{\ell}\left(x_{1}\right) \in \mathbb{Y}$ whereas $f_{\ell}\left(x_{2}\right) \in \partial \mathbb{Y} \backslash \Upsilon_{\ell}$. This leaves us with the only possibility that $x_{1}, x_{2} \notin \mathbb{G}_{\ell}$, so $y \in \partial \mathbb{Y} \backslash \Upsilon_{\ell}$, contradicting Claim 2.

All that remains is to prove Proposition 5.1. Some additional prerequisites, more specific to the proof of Proposition 5.1, are in order.

\subsection{Squares and cells}

Let $\widehat{h}: \widehat{\mathbb{C}} \stackrel{\text { onto }}{\longrightarrow} \widehat{\mathbb{C}}$ be an arbitrary continuous monotone map, $\widehat{h}: \mathbb{C} \rightarrow \mathbb{C}, \widehat{h}(\infty)=\infty$. Consider an open square $\mathbb{Q} \subset \mathbb{C}$ and its preimage $\Omega=\widehat{h}^{-1}(\mathbb{Q})$. The set $\Omega$ is called a cell, which is surely a domain. Its complement $\widehat{\mathbb{C}} \backslash \Omega$, being equal to $\widehat{h}^{-1}(\widehat{\mathbb{C}} \backslash \mathbb{Q})$, is also connected. In view of unicoherence of $\widehat{\mathbb{C}}$, the boundary $\partial \Omega=\bar{\Omega} \cap(\widehat{\mathbb{C}} \backslash \Omega)$ is connected. Thus $\Omega$ is a simply connected domain. As for the preimage $\widehat{h}^{-1}(\partial \mathbb{Q})$, caution is required. Although it is straightforward that $\partial \widehat{h}^{-1}(\mathbb{Q}) \subset \widehat{h}^{-1}(\partial \mathbb{Q})$, the set $\mathcal{C}:=\widehat{h}^{-1}(\partial \mathbb{Q})$ can be larger than $\partial \widehat{h}^{-1}(\mathbb{Q})$. We note that $\mathcal{C}$ is a continuum disconnecting $\widehat{\mathbb{C}}$ into two components (see Proposition 3.4). Also note that the bounded component of $\mathbb{C} \backslash \mathcal{C}$ equals $\Omega=\widehat{h}^{-1}(\mathbb{Q})$.

Next consider an increasing sequence $\mathbb{Q}_{1} \Subset \mathbb{Q}_{2} \Subset \cdots \Subset \mathbb{Q}$ of open squares, $\mathbb{Q}_{n}=$ $\lambda_{n} \mathbb{Q}, 0<\lambda_{n} \nearrow 1$, so $\bigcup_{n=1}^{\infty} \mathbb{Q}_{n}=\mathbb{Q}$. Hereafter, the notation $\lambda \mathbb{Q}$ stands for the square with the same center as $\mathbb{Q}$ but $\lambda$-times smaller than $\mathbb{Q}$. We will be dealing with the induced cells $\Omega_{1} \Subset \Omega_{2} \Subset \cdots \Subset \Omega$, where $\Omega_{n}=\widehat{h}^{-1}\left(\mathbb{Q}_{n}\right)$, so $\bigcup_{n=1}^{\infty} \Omega_{n}=\Omega$. Choose one of the boundaries $\partial \mathbb{Q}_{n}, n=2,3, \ldots$. This is a Jordan curve which separates $\overline{\mathbb{Q}}_{n-1}$ from $\partial \mathbb{Q}_{n+1}$. By monotonicity of $\widehat{h}: \widehat{\mathbb{C}} \stackrel{\text { onto }}{\longrightarrow} \widehat{\mathbb{C}}$ we see that $C_{n}:=\widehat{h}^{-1}\left(\partial \mathbb{Q}_{n}\right)$ is a continuum in $\Omega_{n+1}$. This continuum separates $\partial \Omega_{n+1}$ from $\bar{\Omega}_{n-1}$. More precisely, $C_{n} \subset \Omega_{n+1}$ and the bounded component of $\widehat{\mathbb{C}} \backslash C_{n}$ contains $\bar{\Omega}_{n-1}$, because no point in $\Omega_{n}=\widehat{h}^{-1}\left(\mathbb{Q}_{n}\right)$ can be connected to $\infty$ by a path in the open set $\widehat{\mathbb{C}} \backslash C_{n}$.

Now suppose we are given a sequence $\left\{\widehat{h}_{j}\right\}_{j=1}^{\infty}$ of continuous monotone mappings $\widehat{h}_{j}: \widehat{\mathbb{C}} \rightarrow \widehat{\mathbb{C}}$ such that $\widehat{h}_{j}: \mathbb{C} \rightarrow \mathbb{C}$ and $\widehat{h}(\infty)=\infty$. We assume that this sequence converges uniformly to $\widehat{h}: \widehat{\mathbb{C}} \rightarrow \widehat{\mathbb{C}}$. Denote $C_{n}^{j}=\widehat{h}_{j}^{-1}\left(\partial \mathbb{Q}_{n}\right), j=1,2, \ldots$ These are continua disconnecting $\widehat{\mathbb{C}}$ into two components. The sets

$$
\Omega_{n}^{j}:=\widehat{h}_{j}^{-1}\left(\mathbb{Q}_{n}\right)
$$

are the bounded components of $\widehat{\mathbb{C}} \backslash C_{n}^{j}$. Just the uniform convergence $\widehat{h}_{j} \rightrightarrows \widehat{h}$ yields

$$
\lim _{j \rightarrow \infty} \sup _{x \in C_{n}^{j}} \operatorname{dist}\left(x, C_{n}\right)=0 .
$$


Therefore, for sufficiently large $j$, say $j \geq j_{n}$, the continuum $C_{n}^{j}$ separates $\bar{\Omega}_{n-1}$ from $\partial \Omega_{n+1}$, which yields the inclusions

$$
\bar{\Omega}_{n-1} \subset \Omega_{n}^{j} \subset \bar{\Omega}_{n}^{j} \subset \Omega_{n+1}, \quad n \geq 2 \text { and } j \geq j_{n} .
$$

5.2.1. Cutting a cell. From now on, $\widehat{h}: \widehat{\mathbb{C}} \rightarrow \widehat{\mathbb{C}}$ will be the continuous monotone extension of $h: \overline{\mathbb{X}} \stackrel{\text { onto }}{\longrightarrow} \mathbb{Y}$, as in Section 3.3. Fix one of the boundary circles $\mathfrak{X}_{1}, \ldots, \mathfrak{X}_{\ell} \subset \partial \mathbb{X}$, say $\mathfrak{X}_{i}$ for some $i=1, \ldots, \ell$, and recall the correspondence $\Upsilon_{i}=h\left(\mathfrak{X}_{i}\right)$ with a boundary circle in $\partial \mathbb{Y}$. We also recall the notation $\mathbb{X}_{i}$ for the reflection of $\mathbb{X}$ across $\mathfrak{X}_{i}$, and $\mathbb{Y}_{i}$ for the reflection of $\mathbb{Y}$ across $\Upsilon_{i}$. Now let an open square $\mathbb{Q}$ intersect $\Upsilon_{i}$ along an open arc $\gamma=\Upsilon_{i} \cap \mathbb{Q}$. We assume that $\mathbb{Q}$ is small enough so it lies entirely in $\mathbb{Y} \cup \Upsilon_{i} \cup \mathbb{Y}_{i}$. The arc $\gamma$ is a cross-cut of $\mathbb{Q}$; it cuts $\mathbb{Q}$ into two connected subdomains $\mathbb{Q} \cap \mathbb{Y}$ and $\mathbb{Q} \cap \mathbb{Y}_{i}$. We shall now look closely at the corresponding cross-cut of the cell $\Omega=\widehat{h}^{-1}(\mathbb{Q})$. The boundary map $h: \mathfrak{X}_{i} \stackrel{\text { onto }}{\longrightarrow} \Upsilon_{i}$, being continuous and monotone, defines an open subarc of $\mathfrak{X}_{i}$,

$$
\beta=\left\{x \in \mathfrak{X}_{i}: h(x) \in \gamma\right\},
$$

which obviously lies in $\Omega$. The endpoints of $\beta$ belong to $\partial \Omega$. Indeed, if $x$ is an endpoint of $\beta$ then there are points $x_{v} \in \beta \subset \Omega$ which converge to $x \notin \beta$, thus $h\left(x_{v}\right) \in \gamma$. Passing to the limit we obtain $h(x) \in \bar{\gamma} \backslash \gamma \subset \partial \mathbb{Q}$. Therefore, $x \notin h^{-1}(\mathbb{Q})=\Omega$, meaning that $x \in \partial \Omega$.

This is surely a geometric folklore that the $\operatorname{arc} \beta \subset \Omega$ (in the simply connected domain $\Omega$ ) whose endpoints lie in $\partial \Omega$ splits $\Omega$ into two connected subdomains, namely,

$$
U=\mathbb{X} \cap \Omega \quad \text { and } \quad \mathbb{X}_{i} \cap \Omega .
$$

The latter subdomain will be of no interest to us. Be aware that in general $U$ is not the same as $h^{-1}(\mathbb{Q} \cap \mathbb{Y})$. In fact, we have obtained the domain

$$
U=h^{-1}(\mathbb{Q} \cap \overline{\mathbb{Y}})=\{x \in \mathbb{X}: h(x) \in \mathbb{Q} \cap \overline{\mathbb{Y}}\} .
$$

This domain will hereafter be referred to as a cell in $\mathbb{X}$.

We are now approaching the decisive point of the arguments in this paper.

\section{3. $p$-harmonic replacement in a cell (the heart of the matter)}

Here $\mathbb{X}$ and $\mathbb{Y}$ are circular domains and $\mathfrak{X}$ and $\Upsilon$ denote their outer circles. We consider homeomorphisms $\widehat{h}_{j}: \mathbb{X} \stackrel{\text { onto }}{\longrightarrow} \mathbb{Y}$ in $\mathscr{W}^{1, p}\left(\mathbb{X}, \mathbb{R}^{2}\right)$ which take $\mathfrak{X}$ onto $\Upsilon$, and their weak limit $h \in \widehat{\mathscr{H}_{p}}(\mathbb{X}, \mathbb{Y}), \widehat{h}: \mathbb{X} \stackrel{\text { into }}{\longrightarrow} \overline{\mathbb{Y}}$. The domain $U$ in (5.7) will be called an inner cell if $\mathbb{Q} \subset \mathbb{Y}$ and an outer boundary cell if $\mathbb{Q} \cap \partial \mathbb{Y}=\mathbb{Q} \cap \Upsilon \neq \emptyset$.

Proposition 5.3. Let $h \in \widehat{\mathscr{H}}_{p}(\mathbb{X}, \mathbb{Y})$ and $U \subset \mathbb{X}$ be as above. Then there exists $h^{*} \in$ $\widehat{\mathscr{H}_{p}}(\mathbb{X}, \mathbb{Y})$ such that

(i) $h^{*}: U \stackrel{\text { onto }}{\longrightarrow} \mathbb{Q} \cap \mathbb{Y}$ is a p-harmonic diffeomorphism,

(ii) $h^{*}=h: \overline{\mathbb{X}} \backslash U \stackrel{\text { onto }}{\longrightarrow} \overline{\mathbb{Y}} \backslash(\mathbb{Q} \cap \mathbb{Y})$,

(iii) $\iint_{\mathbb{X}}\left|\nabla h^{*}\right|^{p} \leq \iint_{\mathbb{X}}|\nabla h|^{p}$.

Note that (ii) yields $h^{*}=h: \partial \mathbb{X} \stackrel{\text { onto }}{\longrightarrow} \partial \mathbb{Y}$, and so $h^{*} \in h+\mathscr{R}_{\circ}^{p}(\mathbb{X})$. 
Proof. We first construct $h^{*}$ for the inner cell $U=h^{-1}(\mathbb{Q}), \mathbb{Q} \subset \mathbb{Y}$. This highlights the idea and suggests how to modify the arguments to obtain a proof for the boundary cells.

1. Replacement in an inner cell. Consider, as in $\$ 5.2$, an increasing sequence of open squares $\mathbb{Q}_{n}=\lambda_{n} \mathbb{Q}$ and the associated cells in $\mathbb{X}$,

$$
\Omega_{n}=h^{-1}\left(\mathbb{Q}_{n}\right) \subset \mathbb{X}, \quad \Omega_{1} \Subset \Omega_{2} \Subset \cdots \Subset \Omega=h^{-1}(\mathbb{Q}) .
$$

Also recall the continua $C_{n}^{j}=h_{j}^{-1}\left(\partial \mathbb{Q}_{n}\right)$, the domains $\Omega_{n}^{j}=h_{j}^{-1}\left(\mathbb{Q}_{n}\right)$, and the inclusions

$$
\bar{\Omega}_{n-1} \subset \Omega_{n}^{j} \subset \bar{\Omega}_{n}^{j} \subset \Omega_{n+1} \quad \text { for all } j \geq j_{n} \quad(n \geq 2) .
$$

For every $n \geq 2$ we have a homeomorphism

$$
h_{j_{n}}: \Omega_{n}^{j_{n}} \stackrel{\text { onto }}{\longrightarrow} \mathbb{Q}_{n}
$$

of a Jordan domain onto a convex domain. With the aid of Theorem 4.10 we may replace (5.8) by a $p$-harmonic diffeomorphism, which results in the mapping

$$
\widetilde{h}_{n}=\left\{\begin{array}{l}
p \text {-harmonic diffeomorphism of } \Omega_{n}^{j_{n}} \stackrel{\text { onto }}{\longrightarrow} \mathbb{Q}_{n} \\
h_{j_{n}}: \mathbb{X} \backslash \Omega_{n}^{j_{n}} \rightarrow \mathbb{Y} \backslash \mathbb{Q}_{n} .
\end{array}\right.
$$

By Theorem 4.9, we see that $\widetilde{h}_{n} \in \mathscr{H}_{p}(\mathbb{X}, \mathbb{Y})$ and its energy does not increase,

$$
\mathcal{E}_{\mathbb{X}}\left[\tilde{h}_{n}\right] \leq \mathcal{E}_{\mathbb{X}}\left[h_{j_{n}}\right]
$$

Now the desired mapping $h^{*} \in \widehat{\mathscr{H}}_{p}\left(\mathbb{X}, \mathbb{Y}\right.$ ) is a weak limit of $\widetilde{h}_{n}$ (after passing to a subsequence if necessary). We recall that any family of mappings in $\widehat{\mathscr{H}}_{p}(\mathbb{X}, \mathbb{Y})$ whose energy is controlled by a constant is equicontinuous. Therefore, $\widetilde{h}_{n}$ converges uniformly to $h^{*}$ on $\overline{\mathbb{X}}$. For $x \in \overline{\mathbb{X}} \backslash \Omega$, we have $\widetilde{h}_{n}(x)=h_{j_{n}}(x) \rightarrow h(x)$, and so $h^{*}=h$ on $\overline{\mathbb{X}} \backslash \Omega$. Moreover, $h^{*}: \Omega \rightarrow \mathbb{C}$ is $p$-harmonic. From Theorem 4.9 we infer that $h^{*} \in h+\mathscr{W}_{0}^{1, p}(\Omega)$. This theorem also tells us that

$$
\mathcal{E}_{\mathbb{X}}\left[h^{*}\right] \leq \mathcal{E}_{\mathbb{X}}[h] .
$$

It remains to show that $h^{*}: \Omega \stackrel{\text { onto }}{\longrightarrow} \mathbb{Q}$, and it is a diffeomorphism. To this end, fix (temporarily) an arbitrary compactly contained subdomain $\Omega^{\prime} \Subset \Omega$. Note that $\Omega^{\prime} \Subset \Omega_{n}^{j_{n}}$ for sufficiently large $n$. By the $p$-harmonic Hurwitz Theorem 4.13, either the map $h^{*}: \Omega^{\prime} \stackrel{\text { into }}{\longrightarrow}$ $\mathbb{C}$ is a diffeomorphism, or its Jacobian determinant vanishes identically. However, the latter case is easily ruled out by computing the integral of the Jacobian over $\Omega$. Indeed, appealing to (4.10), we see that

$$
\begin{aligned}
\int_{\Omega} J\left(x, h^{*}\right) d x & =\lim _{n \rightarrow \infty} \int_{\Omega} J\left(x, h_{j_{n}}\right) d x \geq \lim _{n \rightarrow \infty} \int_{\Omega_{n}^{j_{n}}} J\left(x, h_{j_{n}}\right) d x \\
& =\lim _{n \rightarrow \infty}\left|\mathbb{Q}_{n}\right|=|\mathbb{Q}|>0 .
\end{aligned}
$$

Hence also $\int_{\Omega^{\prime}} J\left(x, h^{*}\right) d x>0$, at least for all sufficiently large subdomains $\Omega^{\prime} \Subset \Omega$. In conclusion, $h^{*}: \Omega^{\prime} \stackrel{\text { into }}{\longrightarrow} \mathbb{C}$ is a diffeomorphism, and so is $h^{*}: \Omega \stackrel{\text { into }}{\longrightarrow} \mathbb{C}$. Since the homeomorphisms $h_{j_{n}}$ take $\Omega_{n}^{j_{n}} \subset \Omega$ onto $\mathbb{Q}_{n} \subset \mathbb{Q}$ and converge uniformly on $\Omega$ to $h^{*}$, it is an elementary check that $h^{*}: \Omega \stackrel{\text { onto }}{\longrightarrow} \mathbb{Q}$. 
2. Replacement in an outer boundary cell. Although the construction of $h^{*}$ runs along similar lines, there are subtle adjustments necessary to fit the arguments to a cell $U \subset \mathbb{X}$, because it touches the outer boundary $\mathfrak{X} \subset \partial \mathbb{X}$. We denote $\mathbb{X}^{\prime}=\mathbb{X} \cup \mathfrak{X} \cup \varphi(\mathbb{X})$ and $\mathbb{Y}^{\prime}=\mathbb{Y} \cup \Upsilon \cup \psi(\mathbb{Y})$, where $\varphi(\mathbb{X})$ is the reflection of $\mathbb{X}$ across $\mathfrak{X}$, and $\psi(\mathbb{Y})$ is the reflection of $\mathbb{Y}$ across $\Upsilon$. This time $U$ is a portion of $\Omega=\widehat{h}^{-1}(\mathbb{Q}) \subset \mathbb{X}^{\prime}$, whereas $\mathbb{Q}$ is an open square in $\mathbb{Y}^{\prime}$. More precisely, $U=\Omega \cap \mathbb{X}$.

We recall the sequence of homeomorphisms $h_{j}: \mathbb{X} \stackrel{\text { onto }}{\longrightarrow} \mathbb{Y}$ converging to $h$ weakly in $\mathscr{W}^{1, p}\left(\mathbb{X}, \mathbb{R}^{2}\right)$ together with their extensions to continuous monotone mappings $\widehat{h}_{j}: \widehat{\mathbb{C}} \stackrel{\text { onto }}{\longrightarrow} \widehat{\mathbb{C}}$ and $\widehat{h}: \widehat{\mathbb{C}} \stackrel{\text { onto }}{\longrightarrow} \widehat{\mathbb{C}}$. Note that the $\widehat{h}_{j}$ are homeomorphisms in $\mathbb{X}$ as well as in the reflected domain $\varphi(\mathbb{X})$, but not necessarily along the boundary circle. Therefore, this time the mappings

$$
\widehat{h}_{j}: \mathbb{X}^{\prime} \stackrel{\text { onto }}{\longrightarrow} \mathbb{Y}^{\prime}
$$

are only continuous and monotone. Recall from $\S 5.2$ the sequence of open squares $\mathbb{Q}_{n}=$ $\lambda_{n} \mathbb{Q} \subset \mathbb{Y}^{\prime}$, where $0<\lambda_{n} \nearrow 1$, and the cells

$$
\Omega_{n}=\widehat{h}^{-1}\left(\mathbb{Q}_{n}\right) \subset \mathbb{X}^{\prime}, \quad \Omega_{1} \Subset \Omega_{2} \Subset \cdots \Subset \Omega=\widehat{h}^{-1}(\mathbb{Q}) .
$$

Also recall the continua $C_{n}^{j}=\widehat{h}_{j}^{-1}\left(\partial \mathbb{Q}_{n}\right)$, the domains $\Omega_{n}^{j}=\widehat{h}_{j}^{-1}\left(\mathbb{Q}_{n}\right)$, and the inclusions

$$
\bar{\Omega}_{n-1} \subset \Omega_{n}^{j} \subset \bar{\Omega}_{n}^{j} \subset \Omega_{n+1} \quad \text { for all } j \geq j_{n} \quad(n \geq 2) .
$$

Although the continuum $C_{n}^{j}=\widehat{h}_{j}^{-1}\left(\partial \mathbb{Q}_{n}\right)$ may no longer be a Jordan curve, it nevertheless disconnects $\overline{\mathbb{C}}$ into two components; the domain $\Omega_{n}^{j}$ is none other than the bounded component of $\overline{\mathbb{C}} \backslash C_{n}^{j}$. Even the portion $\Omega_{n}^{j} \cap \mathbb{X}$ (always simply connected) may fail to be Jordan. We need to truncate $\Omega_{n}^{j} \cap \mathbb{X}$ slightly near $\mathfrak{X}$ to obtain a Jordan domain compactly contained in $\mathbb{X}$. It will be additionally required that the image of the truncated region under the homeomorphism $h_{j_{n}}$ be convex.

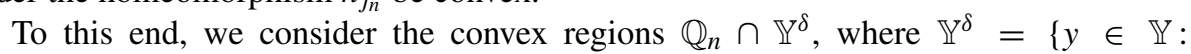
$\operatorname{dist}(y, \partial \mathbb{Y})>\delta\}$. The parameters $\delta=\delta_{n} \rightarrow 0$ must be suitably chosen to ensure that the truncated cells approximate $U$ as $n \rightarrow \infty$.

Let us fix $n \geq 2$ for a moment. Since $h_{j_{n}}: \mathbb{X} \stackrel{\text { onto }}{\longrightarrow} \mathbb{Y}$ is a homeomorphism, there is $\delta=\delta_{n}>0$ such that the set

$$
U_{n}:=h_{j}^{-1}\left(\mathbb{Q}_{n} \cap \mathbb{Y}^{\delta}\right), \quad j=j_{n}, \delta=\delta_{n},
$$

contains $\Omega_{n}^{j} \cap \mathbb{X}^{1 / n}$, where $\mathbb{X}^{1 / n}=\{x \in \mathbb{X}: \operatorname{dist}(x, \partial \mathbb{X})>1 / n\}$.

Now, in analogy with (5.8), we have a sequence of homeomorphisms

$$
h_{j_{n}}: U_{n} \stackrel{\text { onto }}{\longrightarrow} \mathbb{Q}_{n} \cap \mathbb{Y}^{\delta_{n}}
$$

which take the Jordan domains $U_{n}$ onto the convex domains $\mathbb{Q}_{n} \cap \mathbb{Y}^{\delta_{n}}$. As before, we replace $h_{j_{n}}$ by a $p$-harmonic diffeomorphism on $U_{n}$. This furnishes the mapping $\widetilde{h}_{n} \in$ $\widehat{\mathscr{H}}_{p}(\mathbb{X}, \mathbb{Y})$,

$$
\tilde{h}_{n}=\left\{\begin{array}{l}
p \text {-harmonic diffeomorphism of } U_{n} \stackrel{\text { onto }}{\longrightarrow} \mathbb{Q}_{n} \cap \mathbb{Y}^{\delta_{n}} \\
h_{j_{n}}: \mathbb{X} \backslash U_{n} \rightarrow \mathbb{Y} \backslash \mathbb{Q}_{n} \cap \mathbb{Y}^{\delta_{n}}
\end{array}\right.
$$


The desired map $h^{*}$ is a weak limit of $\widetilde{h}_{n}$. The remaining part of the proof goes in the same way as for the inner cell. We leave the details to the interested reader.

\subsection{Proof of Proposition 5.1}

We begin with a geometric covering lemma. For this we recall the neighborhood $\mathbb{Y}^{\prime}=$ $\mathbb{Y} \cup \Upsilon \cup \psi(\mathbb{Y})$ of the outer boundary $\Upsilon \subset \partial \mathbb{Y}$.

Lemma 5.4 (Three nets of squares). Let $\mathbb{Y}^{\prime} \subset \mathbb{R}^{2}$ be any open set. For every $\rho>0$ there exist three nets $\mathcal{A}=\left\{A_{\alpha}\right\}_{\alpha=1}^{\infty}, \mathcal{B}=\left\{B_{\beta}\right\}_{\beta=1}^{\infty}$ and $\mathcal{C}=\left\{C_{\gamma}\right\}_{\gamma=1}^{\infty}$, each consisting of disjoint open squares of diameter $\rho$ or less, such that

$$
\bigcup_{\alpha=1}^{\infty} A_{\alpha} \cup \bigcup_{\beta=1}^{\infty} B_{\beta} \cup \bigcup_{\gamma=1}^{\infty} C_{\gamma}=\mathbb{Y}^{\prime} .
$$

The proof presents no difficulty. We shall not bother the reader with an explicit assembly of the nets. To each of the nets $\mathcal{A}, \mathcal{B}$ and $\mathcal{C}$ there corresponds a reticulation of cells in $\mathbb{X}$. We begin with the net $\mathcal{A}$.

Step 1 ( $p$-harmonic replacements in $\mathcal{A}$-cells). Note that each $A_{\alpha}$ is convex. Consider a disjoint family of open cells in $\mathbb{X}$,

$$
U_{\alpha}=f^{-1}\left(A_{\alpha} \cap \overline{\mathbb{Y}}\right)=\left\{x \in \mathbb{X}: f(x) \in A_{\alpha} \cap \overline{\mathbb{Y}}\right\} .
$$

It should be noted that $U_{\alpha}$ is empty whenever $A_{\alpha} \cap \mathbb{Y}=\emptyset$.

At this stage we appeal to the $p$-harmonic replacements provided by Proposition 5.3, with $U_{\alpha}$ in place of $U$. Upon consecutive replacements in the cells $U_{\alpha}$ we arrive at a mapping $f_{\mathcal{A}}: \overline{\mathbb{X}} \stackrel{\text { onto }}{\longrightarrow} \overline{\mathbb{Y}}$ such that

(i) $f_{\mathcal{A}} \in \widetilde{\mathscr{H}}_{p}(\mathbb{X}, \mathbb{Y})$ and $f_{\mathcal{A}}=f: \partial \mathbb{X} \stackrel{\text { onto }}{\longrightarrow} \partial \mathbb{Y}$,

(ii) $f_{\mathcal{A}}: \bigcup_{\alpha=1}^{\infty} U_{\alpha} \stackrel{\text { onto }}{\longrightarrow} \bigcup_{\alpha=1}^{\infty}\left(A_{\alpha} \cap \mathbb{Y}\right)$ is a $p$-harmonic diffeomorphism,

(iii) $f_{\mathcal{A}}=f: \overline{\mathbb{X}} \backslash \bigcup_{\alpha=1}^{\infty} U_{\alpha} \stackrel{\text { onto }}{\longrightarrow} \overline{\mathbb{Y}} \backslash \bigcup_{\alpha=1}^{\infty}\left(A_{\alpha} \cap \mathbb{Y}\right)$,

(iv) $\iint_{\mathbb{X}}\left|\nabla f_{\mathcal{A}}\right|^{p} \leq \iint_{\mathbb{X}}|\nabla f|^{p}$.

The net $\mathcal{A}$ actually depends on $\rho>0$, which we indicate by writing $\mathcal{A}=\mathcal{A}_{\rho}$. Let us take a close look at the mappings $f_{\mathcal{A}_{\rho}}$ when $\rho$ approaches 0 . First, given $x \in \mathbb{X}$, we see that $f_{\mathcal{A}_{\rho}}(x)=f(x)$ if $x$ does not belong to any of the cells $U_{\alpha}$. If, however, $x \in U_{\alpha}$ for some $\alpha$ then

$$
f_{\mathcal{A}_{\rho}}(x) \in f\left(U_{\alpha}\right)=A_{\alpha} \cap \overline{\mathbb{Y}} .
$$

In either case $\left|f_{\mathcal{A}_{\alpha}}(x)-f(x)\right| \leq \rho$. We then infer that $\left\|f_{\mathcal{A}_{\rho}}-f\right\|_{\mathscr{C}(\mathbb{X})} \leq \rho$, which means that $f_{\mathcal{A}_{\delta}} \rightrightarrows f$ (uniformly on $\mathbb{X}$ ) as $\rho \rightarrow 0$. On the other hand, the energy of $f_{\mathcal{A}_{\delta}}$ does not exceed that of $f$. Thus also $f_{\mathcal{A}_{\delta}} \rightarrow f$ weakly in $\mathscr{W}^{1, p}(\mathbb{X})$. Hence, by lower semicontinuity of the energy functional,

$$
\mathcal{E}_{\mathbb{X}}[f] \leq \lim _{\rho \rightarrow 0} \mathcal{E}_{\mathbb{X}}\left[f_{\mathcal{A}_{\rho}}\right] \leq \lim _{\rho \rightarrow 0} \mathcal{E}_{\mathbb{X}}[f]=\mathcal{E}_{\mathbb{X}}[f], \quad \text { so } \quad \lim _{\rho \rightarrow 0} \mathcal{E}_{\mathbb{X}}\left[f_{\mathcal{A}_{\rho}}\right]=\mathcal{E}_{\mathbb{X}}[f]
$$


Since the space $\mathscr{L}^{p}(\mathbb{X})$ is uniformly convex, the mappings $f_{\mathcal{A}_{\delta}}$ actually converge strongly in $\mathscr{R}^{p}(\mathbb{X})$.

In summary, given any $\epsilon>0$, there is a net $\mathcal{A}=\left\{A_{\alpha}\right\}_{\alpha=1}^{\infty}$ for which

$$
\left\|h_{\mathcal{A}}-h\right\|_{\mathscr{R} p(\mathbb{X})} \leq \frac{\epsilon}{3 \ell}
$$

Step 2 ( $p$-harmonic replacements in $\mathcal{B}$-cells). This is essentially a repetition of the procedure in Step 1, but with $f_{\mathcal{A}}$ in place of $f$ and with the net $\mathcal{B}$ in place of $\mathcal{A}$. It gives us another reticulation of open cells in $\mathbb{X}$. Namely, we obtain

$$
V_{\beta}=f_{\mathcal{A}}^{-1}\left(B_{\beta} \cap \overline{\mathbb{Y}}\right)=\left\{x \in \mathbb{X}: f_{\mathcal{A}}(x) \in B_{\beta} \cap \overline{\mathbb{Y}}\right\}
$$

and a mapping $f_{\mathcal{A B}}: \overline{\mathbb{X}} \stackrel{\text { onto }}{\longrightarrow} \overline{\mathbb{Y}}$, such that

(i) $f_{\mathcal{A B}} \in \widehat{\mathscr{H}}_{p}(\mathbb{X}, \mathbb{Y})$ and $f_{\mathcal{A B}}=f_{\mathcal{A}}=f: \partial \mathbb{X} \stackrel{\text { onto }}{\longrightarrow} \partial \mathbb{Y}$,

(ii) $f_{\mathcal{A B}}: \bigcup_{\beta=1}^{\infty} V_{\beta} \stackrel{\text { onto }}{\longrightarrow} \bigcup_{\beta=1}^{\infty}\left(B_{\beta} \cap \mathbb{Y}\right)$ is a $p$-harmonic diffeomorphism,

(iii) $f_{\mathcal{A B}}=f_{\mathcal{A}}: \overline{\mathbb{X}} \backslash \bigcup_{\beta=1}^{\infty} V_{\beta} \stackrel{\text { onto }}{\longrightarrow} \overline{\mathbb{Y}} \backslash \bigcup_{\beta=1}^{\infty}\left(B_{\beta} \cap \mathbb{Y}\right)$,

(iv) $\iint_{\mathbb{X}}\left|\nabla f_{\mathcal{A B}}\right|^{p} \leq \iint_{\mathbb{X}}\left|\nabla f_{\mathcal{A}}\right|^{p} \leq \iint_{\mathbb{X}}|\nabla f|^{p}$.

The counterpart of (5.12) reads

$$
\left\|f_{\mathcal{A B}}-f_{\mathcal{A}}\right\|_{\mathscr{R}}{ }_{(\mathbb{X})} \leq \frac{\epsilon}{3 \ell}
$$

Step 3 ( $p$-harmonic replacements in $\mathcal{C}$-cells). Analogously to Steps 1 and 2, we assemble open cells in $X$ associated with the net $\mathcal{C}$,

$$
W_{\gamma}=f_{\mathcal{A B}}^{-1}\left(C_{\gamma} \cap \overline{\mathbb{Y}}\right)=\left\{x \in \mathbb{X}: f_{\mathcal{A B}}(x) \in C_{\gamma} \cap \overline{\mathbb{Y}}\right\}
$$

The $p$-harmonic replacements in these cells result in the mapping, denoted by $f_{\mathcal{A B C}}$ : $\overline{\mathbb{X}} \stackrel{\text { onto }}{\longrightarrow} \overline{\mathbb{Y}}$, such that

(i) $f_{\mathcal{A B C}} \in \widetilde{\mathscr{H}}_{p}(\mathbb{X}, \mathbb{Y})$ and $f_{\mathcal{A B C}}=f_{\mathcal{A B}}=h_{\mathcal{A}}=f: \partial \mathbb{X} \stackrel{\text { onto }}{\longrightarrow} \partial \mathbb{Y}$,

(ii) $f_{\mathcal{A B C}}: \bigcup_{\gamma=1}^{\infty} W_{\gamma} \stackrel{\text { onto }}{\longrightarrow} \bigcup_{\beta=1}^{\infty}\left(C_{\gamma} \cap \mathbb{Y}\right)$ is a $p$-harmonic diffeomorphism,

(iii) $f_{\mathcal{A B C}}=f_{\mathcal{A B}}: \overline{\mathbb{X}} \backslash \bigcup_{\gamma=1}^{\infty} W_{\gamma} \stackrel{\text { onto }}{\longrightarrow} \overline{\mathbb{Y}} \backslash \bigcup_{\beta=1}^{\infty}\left(C_{\gamma} \cap \mathbb{Y}\right)$,

(iv) $\iint_{\mathbb{X}}\left|\nabla f_{\mathcal{A B C}}\right|^{p} \leq \iint_{\mathbb{X}}\left|\nabla f_{\mathcal{A B}}\right|^{p} \leq \iint_{\mathbb{X}}|\nabla f|^{p}$.

Moreover,

$$
\left\|f_{\mathcal{A B C}}-f_{\mathcal{A B}}\right\|_{\mathscr{R}} p(\mathbb{X}) \leq \frac{\epsilon}{3 \ell}
$$


5.4.1. The desired mapping $f^{*}$. Having performed the replacement procedures in the $\mathcal{A}$ cells, $\mathcal{B}$-cells and $\mathcal{C}$-cells we finally arrive at the mapping $f_{\mathcal{A B C}}$. We allege that this map satisfies all the conditions asserted in Proposition 5.1, whence we denote it by

$$
f^{*}=f_{\mathcal{A B C}} \in \widehat{\mathscr{H}}_{p}(\mathbb{X}, \mathbb{Y}) \text {. }
$$

Let us verify those conditions. Inequality (5.2) is obvious;

$$
\begin{aligned}
\left\|f^{*}-f\right\|_{\mathscr{R} p(\mathbb{X})} & \leq\left\|f_{\mathcal{A B C}}-f_{\mathcal{A B}}\right\|_{\mathscr{R} p(\mathbb{X})}+\left\|f_{\mathcal{A B}}-f_{\mathcal{A}}\right\|_{\mathscr{R} p(\mathbb{X})}+\left\|f_{\mathcal{A}}-f\right\|_{\mathscr{R} p(\mathbb{X})} \\
& \leq \frac{\epsilon}{3 \ell}+\frac{\epsilon}{3 \ell}+\frac{\epsilon}{3 \ell}=\frac{\epsilon}{\ell} .
\end{aligned}
$$

Verification of the other conditions involves elementary set-theoretical considerations, which we include for completeness; some do not seem immediate at all. First we show the identity

$$
\bigcup_{\alpha=1}^{\infty} U_{\alpha} \cup \bigcup_{\beta=1}^{\infty} V_{\beta} \cup \bigcup_{\gamma=1}^{\infty} W_{\gamma}=\mathbb{G}
$$

where we recall that $\mathbb{G}=\{x \in \mathbb{X}: f(x) \in \mathbb{Y} \cup \Upsilon\}$.

Proof of (5.14). Suppose $x \in \bigcup_{\alpha=1}^{\infty} U_{\alpha} \cup \bigcup_{\beta=1}^{\infty} V_{\beta} \cup \bigcup_{\gamma=1}^{\infty} W_{\gamma}$. There are three cases to consider.

Case 1: $x \in \bigcup_{\alpha=1}^{\infty} U_{\alpha}$. This yields $h(x) \in A_{\alpha} \cap \overline{\mathbb{Y}} \subset \mathbb{Y} \cup \Upsilon$ for some $\alpha$. Thus $x \in \mathbb{G}$.

Case 2: $x \notin \bigcup_{\alpha=1}^{\infty} U_{\alpha}$ and $x \in \bigcup_{\beta=1}^{\infty} V_{\beta}$. Hence for some $\beta$,

$$
f(x)=f_{\mathcal{A}}(x) \in B_{\beta} \cap \overline{\mathbb{Y}} \subset \mathbb{Y} \cup \Upsilon, \quad \text { thus } x \in \mathbb{G} .
$$

Case 3: $x \notin \bigcup_{\alpha=1}^{\infty} U_{\alpha}$ and $x \notin \bigcup_{\beta=1}^{\infty} V_{\beta}$, so $x \in W_{\gamma}$ for some $\gamma$. This yields

$$
f(x)=f_{\mathcal{A}}(x)=f_{\mathcal{A B}}(x) \in C_{\gamma} \cap \overline{\mathbb{Y}} \subset \mathbb{Y} \cup \Upsilon, \quad \text { thus } x \in \mathbb{G} .
$$

In either case we have the inclusion $\bigcup_{\alpha=1}^{\infty} U_{\alpha} \cup \bigcup_{\beta=1}^{\infty} V_{\beta} \cup \bigcup_{\gamma=1}^{\infty} W_{\gamma} \subset \mathbb{G}$.

For the opposite inclusion, suppose that $x \in \mathbb{X}$ and

$$
x \notin \bigcup_{\alpha=1}^{\infty} U_{\alpha} \cup \bigcup_{\beta=1}^{\infty} V_{\beta} \cup \bigcup_{\gamma=1}^{\infty} W_{\gamma} .
$$

Since $x \notin \bigcup_{\alpha=1}^{\infty} U_{\alpha}$, we see that $f(x)=f_{\mathcal{A}}(x)$. Since $x \notin \bigcup_{\beta=1}^{\infty} V_{\beta}$, we see that $f_{\mathcal{A}}(x)=f_{\mathcal{A B}}(x)$. Since $x \notin \bigcup_{\gamma=1}^{\infty} W_{\gamma}$, we see that $f_{\mathcal{A B}}(x)=f_{\mathcal{A B C}}(x)=f^{*}(x)$. Consider the point $y=f(x)=f_{\mathcal{A}}(x)=f_{\mathcal{A B}}(x)=f_{\mathcal{A B C}}(x)$. We have

$y=f(x) \notin \bigcup_{\alpha=1}^{\infty}\left(A_{\alpha} \cap \overline{\mathbb{Y}}\right), \quad y=f_{\mathcal{A}}(x) \notin \bigcup_{\beta=1}^{\infty}\left(B_{\beta} \cap \overline{\mathbb{Y}}\right), \quad y=f_{\mathcal{A B}}(x) \notin \bigcup_{\gamma=1}^{\infty}\left(C_{\gamma} \cap \overline{\mathbb{Y}}\right)$.

Thus

$$
y \notin\left(\bigcup_{\alpha=1}^{\infty} A_{\alpha} \cup \bigcup_{\beta=1}^{\infty} B_{\beta} \cup \bigcup_{\gamma=1}^{\infty} C_{\gamma}\right) \cap \overline{\mathbb{Y}}=\mathbb{Y} \cup \Upsilon .
$$


The last equality is immediate from (5.11). Thus $h(x) \notin \mathbb{Y} \cup \Upsilon$, and so $x \notin \mathbb{G}$. This completes the proof of (5.14).

In order to see that $f^{*}: \mathbb{G} \rightarrow \mathbb{Y}$ is injective, we consider distinct points

$$
x_{1}, x_{2} \in \mathbb{G}=\bigcup_{\alpha=1}^{\infty} U_{\alpha} \cup \bigcup_{\beta=1}^{\infty} V_{\beta} \cup \bigcup_{\gamma=1}^{\infty} W_{\gamma} .
$$

Suppose, to the contrary, that $f^{*}\left(x_{1}\right)=f^{*}\left(x_{2}\right)=: y$. Since the mapping $f^{*}=$ $f_{\mathcal{A B C}}: \bigcup_{\gamma=1}^{\infty} W_{\gamma} \stackrel{\text { onto }}{\longrightarrow} \bigcup_{\gamma=1}^{\infty}\left(C_{\gamma} \cap \mathbb{Y}\right)$ is injective and

$$
f_{\mathcal{A B C}}=f_{\mathcal{A B}}: \mathbb{X} \backslash \bigcup_{\gamma=1}^{\infty} W_{\gamma} \rightarrow \overline{\mathbb{Y}} \backslash \bigcup_{\gamma=1}^{\infty}\left(C_{\gamma} \cap \mathbb{Y}\right)
$$

it follows that $x_{1}, x_{2} \notin \bigcup_{\gamma=1}^{\infty} W_{\gamma}$. Thus we are reduced to the case $x_{1}, x_{2} \in \bigcup_{\beta=1}^{\infty} U_{\alpha} \cup$ $\bigcup_{\beta=1}^{\infty} V_{\beta}$ and $y=f_{\mathcal{A B}}\left(x_{1}\right)=f_{\mathcal{A B}}\left(x_{2}\right)$. In exactly the same way we infer that $x_{1}, x_{2} \notin$ $\bigcup_{\beta=1}^{\infty} V_{\beta}$. Thus we are further reduced to the case

$$
x_{1}, x_{2} \in \bigcup_{\alpha=1}^{\infty} U_{\alpha} \quad \text { and } \quad y=f_{\mathcal{A}}\left(x_{1}\right)=f_{\mathcal{A}}\left(x_{2}\right) .
$$

Just as before, $x_{1}, x_{2} \notin \bigcup_{\alpha=1}^{\infty} U_{\alpha}$. We arrive at the contradiction

$$
x_{1}, x_{2} \notin \bigcup_{\alpha=1}^{\infty} U_{\alpha} \cup \bigcup_{\beta=1}^{\infty} V_{\beta} \cup \bigcup_{\gamma=1}^{\infty} W_{\gamma}=\mathbb{G},
$$

finishing the argument for injectivity of $f^{*}: \mathbb{G} \rightarrow \mathbb{Y}$.

Next, to see that $f^{*}: \mathbb{G} \stackrel{\text { into }}{\longrightarrow} \mathbb{Y}$, we choose

$$
x \in \mathbb{G}=\bigcup_{\alpha=1}^{\infty} U_{\alpha} \cup \bigcup_{\beta=1}^{\infty} V_{\beta} \cup \bigcup_{\gamma=1}^{\infty} W_{\gamma} .
$$

Denote $y=f^{*}(x)$. If $x \in \bigcup_{\gamma=1}^{\infty} W_{\gamma}$, then

$$
f^{*}(x)=f_{\mathcal{A B C}}(x) \in \bigcup_{\gamma=1}^{\infty} C_{\gamma} \cap \mathbb{Y} \subset \mathbb{Y} .
$$

Thus suppose $x \notin \bigcup_{\gamma=1}^{\infty} W_{\gamma}$. In this case $f^{*}(x)=f_{\mathcal{A B}}(x)$ and $x \in \bigcup_{\alpha=1}^{\infty} U_{\alpha} \cup \bigcup_{\beta=1}^{\infty} V_{\beta}$. Now, if $x \in \bigcup_{\beta=1}^{\infty} V_{\beta}$ then $y=f_{\mathcal{A B}} \in \bigcup_{\beta=1}^{\infty} B_{\beta} \cap \mathbb{Y} \subset \mathbb{Y}$, as desired. Finally, if $x \notin \bigcup_{\beta=1}^{\infty} V_{\beta}$ then $x \in \bigcup_{\beta=1}^{\infty} U_{\alpha}$, which yields $y=f_{\mathcal{A}}(x) \in \bigcup_{\alpha=1}^{\infty} A_{\alpha} \cap \mathbb{Y} \subset \mathbb{Y}$, completing the proof of the inclusion $f^{*}(\mathbb{G}) \subset \mathbb{Y}$.

Lastly, to see surjectivity $f^{*}: \mathbb{G} \stackrel{\text { onto }}{\longrightarrow} \mathbb{Y}$ we recall that $f^{*}=f_{\mathcal{A B C}}: \overline{\mathbb{X}} \stackrel{\text { onto }}{\longrightarrow} \overline{\mathbb{Y}}$ and $f^{*}=f: \partial \mathbb{X} \stackrel{\text { onto }}{\longrightarrow} \partial \mathbb{Y}$. Hence $f^{*}(\mathbb{X}) \supset \mathbb{Y}$. Take any $y \in \mathbb{Y}$ to show that it lies in 
$f^{*}(\mathbb{G})$. Obviously, there exists $x \in \mathbb{X}$ such that $f^{*}(x)=y$, whereas the assumption $x \notin \mathbb{G}=\bigcup_{\alpha=1}^{\infty} U_{\alpha} \cup \bigcup_{\beta=1}^{\infty} V_{\beta} \cup \bigcup_{\gamma=1}^{\infty} W_{\gamma}$ leads to a clear contradiction,

$$
y=f^{*}(x)=f_{\mathcal{A B C}}(x)=f_{\mathcal{A B}}(x)=f_{\mathcal{A}}(x)=f(x) \in \mathbb{Y}, \quad \text { so } \quad x \in f^{-1}(\mathbb{Y}) \subset \mathbb{G} .
$$

finishing the proof of Proposition 5.1.

This also completes the proof of Theorem 1.2.

Acknowledgments. T. Iwaniec was supported by the NSF grant DMS-1301558 and the Academy of Finland project 1128331. J. Onninen was supported by the NSF grant DMS- 1301570.

\section{References}

[1] Alessandrini, G.: Critical points of solutions of elliptic equations in two variables. Ann. Scuola Norm. Sup. Pisa Cl. Sci. (4) 14, 229-256 (1987) Zbl 0649.35026 MR 0939628

[2] Alessandrini, G., Nesi, V.: Univalent $\sigma$-harmonic mappings. Arch. Ration. Mech. Anal. 158, 155-171 (2001) Zbl 0977.31006 MR 1838656

[3] Alessandrini, G., Sigalotti, M.: Geometric properties of solutions to the anisotropic pLaplace equation in dimension two. Ann. Acad. Sci. Fenn. Math. 26, 249-266 (2001) Zbl 1002.35044 MR 1816571

[4] Antman, S. S.: Nonlinear Problems of Elasticity. Appl. Math. Sci. 107, Springer, New York (1995) Zbl 0820.73002 MR 1323857

[5] Astala, K., Iwaniec, T., Martin, G.: Elliptic Partial Differential Equations and Quasiconformal Mappings in the Plane. Princeton Univ. Press, Princeton, NJ (2009) Zbl 1182.30001 MR 2472875

[6] Astala, K., Iwaniec, T., Martin, G.: Deformations of annuli with smallest mean distortion. Arch. Ration. Mech. Anal. 195, 899-921 (2010) Zbl 1219.30011 MR 2591976

[7] Astala, K., Iwaniec, T., Saksman, E.: Beltrami operators in the plane. Duke Math. J. 107, 27-56 (2001) Zbl 1009.30015 MR 1815249

[8] Ball, J. M.: Convexity conditions and existence theorems in nonlinear elasticity. Arch. Ration. Mech. Anal. 63, 337-403 (1976/77) Zbl 0368.73040 MR 0475169

[9] Ball, J. M.: Constitutive inequalities and existence theorems in nonlinear elastostatics. In: Nonlinear Analysis and Mechanics: Heriot-Watt Symposium (Edinburgh, 1976), Vol. I, Res. Notes Math. 17, Pitman, London, 187-241 (1977) Zbl 03588136 MR 0478899

[10] Ball, J. M.: Existence of solutions in finite elasticity. In: Finite Elasticity (Betlehem, PA, 1980), Nijhoff, 1-12 (1982) Zbl 0518.73031

[11] Ball, J. M.: Discontinuous equilibrium solutions and cavitation in nonlinear elasticity. Philos. Trans. Roy. Soc. London A 306, 557-611 (1982) Zbl 0513.73020 MR 0703623

[12] Ball, J. M.: Minimizers and the Euler-Lagrange equations. In: Trends and Applications of Pure Mathematics to Mechanics (Palaiseau, 1983), Lecture Notes in Phys. 195, Springer, Berlin, 1-4 (1984) Zbl 0547.73013 MR 0755716

[13] Ball, J. M.: Some open problems in elasticity. In: Geometry, Mechanics, and Dynamics, Springer, New York, 3-59 (2002) Zbl 1054.74008 MR 1919825

[14] Bauman, P., Marini, A., Nesi, V.: Univalent solutions of an elliptic system of partial differential equations arising in homogenization. Indiana Univ. Math. J. 50, 747-757 (2001) Zbl 1330.35121 MR 1871388 
[15] Bauman, P., Owen, N. C., Phillips, D.: Maximum principles and a priori estimates for an incompressible material in nonlinear elasticity. Comm. Partial Differential Equations 17, 11851212 (1992) Zbl 0777.35014 MR 1179283

[16] Bojarski, B.: Generalized solutions of a system of differential equations of first order and of elliptic type with discontinuous coefficients. Mat. Sb. (N.S.) 43 (85), 451-503 (1957) Zbl 1173.35403 MR 0106324

[17] Bojarski, B., Iwaniec, T.: $p$-harmonic equation and quasiregular mappings. In: Partial Differential Equations (Warszawa, 1984), Banach Center Publ. 19, PWN, Warszawa, 25-38 (1987) Zbl 0659.35035 MR 1055157

[18] Ciarlet, P. G.: Mathematical Elasticity Vol. I. Three-Dimensional Elasticity. North-Holland Stud. Math. Appl. 20, North-Holland, Amsterdam (1988) Zbl 0648.73014 MR 0936420

[19] Coifman, R., Lions, P.-L., Meyer, Y., Semmes, S.: Compensated compactness and Hardy spaces. J. Math. Pures Appl. (9) 72, 247-286 (1993) Zbl 0864.42009 MR 1225511

[20] Conti, S., De Lellis, C.: Some remarks on the theory of elasticity for compressible Neohookean materials. Ann. Scuola Norm. Sup. Pisa Cl. Sci. (5) 2, 521-549 (2003) Zbl 1114.74004 MR 2020859

[21] Cristina, J., Iwaniec, T., Kovalev, L. V., Onninen, J.: The Hopf-Laplace equation: harmonicity and regularity. Ann. Scuola Norm. Sup. Pisa Cl. Sci. (5) 13, 1145-1187 (2014) Zbl 1327.30022 MR 3362123

[22] D'Onofrio, L., Iwaniec, T.: Notes on $p$-harmonic analysis. In: The $p$-harmonic Equation and Recent Advances in Analysis, Contemp. Math. 370, Amer. Math. Soc., Providence, RI, 25-49 (2005) Zbl 1134.35332 MR 2126700

[23] Duren, P.: Harmonic Mappings in the Plane. Cambridge Tracts in Math. 156, Cambridge Univ. Press, Cambridge (2004) Zbl 1055.31001 MR 2048384

[24] Evans, L. C.: Quasiconvexity and partial regularity in the calculus of variations. Arch. Ration. Mech. Anal. 95, 227-252 (1986) Zbl 0627.49006 MR 0853966

[25] Hajłasz, P.: Pointwise Hardy inequalities. Proc. Amer. Math. Soc. 127, 417-423 (1999) Zbl 0911.31005 MR 1458875

[26] Hajłasz, P.: Sobolev mappings: Lipschitz density is not a bi-Lipschitz invariant of the target. Geom. Funct. Anal. 17, 435-467 (2007) Zbl 1132.46023 MR 2322491

[27] Heinonen, J., Kilpeläinen, T., Malý, J.: Connectedness in fine topologies. Ann. Acad. Sci. Fenn. Ser. A I Math. 15, 107-123 (1990) Zbl 0715.31005 MR 1050785

[28] Heinonen, J., Kilpeläinen, T., Martio, O.: Nonlinear Potential Theory of Degenerate Elliptic Equations. Oxford Univ. Press, New York (1993) Zbl 0780.31001 MR 1207810

[29] Iwaniec, T., Koh, N.-T., Kovalev, L. V., Onninen, J.: Existence of energy-minimal diffeomorphisms between doubly connected domains. Invent. Math. 186, 667-707 (2011) Zbl 1255.30031 MR 2854087

[30] Iwaniec, T., Kovalev, L. V., Onninen, J.: Diffeomorphic approximation of Sobolev homeomorphisms. Arch. Ration. Mech. Anal. 201, 1047-1067 (2011) Zbl 1260.46023 MR 2824471

[31] Iwaniec, T., Kovalev, L. V., Onninen, J.: Approximation up to the boundary of homeomorphisms of finite Dirichlet energy. Bull. London Math. Soc. 44, 871-881 (2012) Zbl 1264.30025 MR 2975148

[32] Iwaniec, T., Kovalev, L. V., Onninen, J.: Lipschitz regularity for inner-variational equations. Duke Math. J. 162, 643-672 (2013) Zbl 1319.49055 MR 3039677

[33] Iwaniec, T., Manfredi, J. J.: Regularity of $p$-harmonic functions on the plane. Rev. Mat. Iberoamer. 5, 1-19 (1989) Zbl 0805.31003 MR 1057335

[34] Iwaniec, T., Martin, G.: Geometric Function Theory and Non-linear Analysis. Oxford Math. Monogr., Oxford Univ. Press (2001) Zbl 1045.30011 MR 1859913 
[35] Iwaniec, T., Onninen, J.: $\mathcal{H}^{1}$-estimates of Jacobians by subdeterminants. Math. Ann. 324, 341-358 (2002) Zbl 1055.42011 MR 1933861

[36] Iwaniec, T., Onninen, J.: Hyperelastic deformations of smallest total energy. Arch. Ration. Mech. Anal. 194, 927-986 (2009) Zbl 1193.74013 MR 2563629

[37] Iwaniec, T., Onninen, J.: Neohookean deformations of annuli, existence, uniqueness and radial symmetry. Math. Ann. 348, 35-55 (2010) Zbl 1214.30011 MR 2657433

[38] Iwaniec, T., Onninen, J.: Deformations of finite conformal energy: existence and removability of singularities. Proc. London Math. Soc. (3) 100, 1-23 (2010) Zbl 1196.30023 MR 2578466

[39] Iwaniec, T., Onninen, J.: Deformations of finite conformal energy: boundary behavior and limit theorems. Trans. Amer. Math. Soc. 363, 5605-5648 (2011) Zbl 1246.30040 MR 2817402

[40] Iwaniec, T., Onninen, J.: $n$-Harmonic mappings between annuli: the art of integrating free Lagrangians. Mem. Amer. Math. Soc. 218, no. 1023 (2012) Zbl 1294.30042 MR 2976798

[41] Iwaniec, T., Onninen, J.: Mappings of least Dirichlet energy and their Hopf differentials. Arch. Ration. Mech. Anal. 209, 401-453 (2013) Zbl 1294.30085 MR 3056614

[42] Iwaniec, T., Onninen, J.: Monotone Sobolev mappings of planar domains and surfaces. Arch. Ration. Mech. Anal. 219, 159-181 (2016) Zbl 06545481 MR 3437849

[43] Jerison, D. S., Kenig, C. E.: Hardy spaces, $A_{\infty}$, and singular integrals on chord-arc domains. Math. Scand. 50, 221-247 (1982) Zbl 0509.30025 MR 0672926

[44] Jost, J.: A note on harmonic maps between surfaces. Ann. Inst. H. Poincaré Anal. Non Linéaire 2, 397-405 (1985) Zbl 0585.58011 MR 0831039

[45] Jost, J.: Two-Dimensional Geometric Variational Problems. Wiley, Chichester (1991) Zbl 0729.49001 MR 1100926

[46] Jost, J., Schoen, R.: On the existence of harmonic diffeomorphisms. Invent. Math. 66, 353359 (1982) Zbl 0488.58009 MR 0656629

[47] Kilpeläinen, T., Malý, J.: Degenerate elliptic equations with measure data and nonlinear potentials. Ann. Scuola Norm. Sup. Pisa Cl. Sci. (4) 19, 591-613 (1992) Zbl 0797.35052 MR 1205885

[48] Kuratowski, K.: On the completeness of the space of monotone mappings and some related problems. Bull. Acad. Polon. Sci. Sér. Sci. Math. Astronom. Phys. 16, 283-285 (1968) Zbl 0155.50201 MR 0232337

[49] Kuratowski, K., Lacher, R. C.: A theorem on the space of monotone mappings. Bull. Acad. Polon. Sci. Sér. Sci. Math. Astronom. Phys. 17, 797-800 (1969) Zbl 0195.24401 MR 0275386

[50] Lehrbäck, J.: Pointwise Hardy inequalities and uniformly fat sets. Proc. Amer. Math. Soc. 136, 2193-2200 (2008) Zbl 1151.46021 MR 2383525

[51] Lehto, O., Virtanen, K. I.: Quasiconformal Mappings in the Plane. Springer, New York (1973) Zbl 0267.30016 MR 0344463

[52] Lewis, J. L.: On critical points of $p$-harmonic functions in the plane. Electron. J. Differential Equations 1994, no. 3, 4 pp. Zbl 0808.31008 MR 1281471

[53] Manfredi, J. J.: $p$-harmonic functions in the plane. Proc. Amer. Math. Soc. 103, 473-479 (1988) Zbl 0658.35041 MR 0943069

[54] Marcus, M., Mizel, V. J.: Every superposition operator mapping one Sobolev space into another is continuous. J. Funct. Anal. 33, 217-229 (1979) Zbl 0418.46024 MR 0546508

[55] Marsden, J. E., Hughes, T. J. R.: Mathematical Foundations of Elasticity. Dover Publ., New York (1994) MR 1262126 
[56] McAuley, L. F.: Some fundamental theorems and problems related to monotone mappings. In: Proc. First Conf. on Monotone Mappings and Open Mappings (Binghamton, NY, 1970), State Univ. of New York at Binghamton, NY, 1-36 (1971) Zbl 0226.54006 MR 0287518

[57] Miniowitz, R.: Normal families of quasimeromorphic mappings. Proc. Amer. Math. Soc. 84, 35-43 (1982) Zbl 0478.30024 MR 0633273

[58] Morrey, C. B.: The topology of (path) surfaces. Amer. J. Math. 57, 17-50 (1935) Zbl 0011.03701 MR 1507053

[59] Morrey, C. B.: Quasi-convexity and the lower semicontinuity of multiple integrals. Pacific J. Math. 2, 25-53 (1952) Zbl 0046.10803 MR 0054865

[60] Müller, S.: Higher integrability of determinants and weak convergence in $L^{1}$. J. Reine Angew. Math. 412, 20-34 (1990) Zbl 0713.49004 MR 1078998

[61] Radó, T.: On continuous mappings of Peano spaces. Trans. Amer. Math. Soc. 58, 420-454 (1945) Zbl 0061.10802 MR 0014419

[62] Radó, T.: Length and Area. Amer. Math. Soc., New York (1948) Zbl 0033.17002 MR 0024511

[63] Reshetnyak, Yu. G.: Space Mappings with Bounded Distortion. Amer. Math. Soc., Providence, RI (1989) Zbl 0667.30018 MR 0994644

[64] Šilhavý, M.: The Mechanics and Thermodynamics of Continuous Media. Texts Monogr. Phys., Springer, Berlin (1997) Zbl 0870.73004 MR 1423807

[65] Sivaloganathan, J., Spector, S. J.: Necessary conditions for a minimum at a radial cavitating singularity in nonlinear elasticity. Ann. Inst. H. Poincaré Anal. Non Linéaire 25, 201-213 (2008) Zbl 1137.74011 MR 2383087

[66] Sivaloganathan, J., Spector, S. J.: On irregular weak solutions of the energy-momentum equations. Proc. Roy. Soc. Edinburgh Sect. A 141, 193-204 (2011) Zbl 1211.49006 MR 2773446

[67] Truesdell, C., Noll, W.: The Non-linear Field Theories of Mechanics. Springer, Berlin (2004) Zbl 0779.73004 MR 2056350

[68] Tukia, P.: The planar Schönflies theorem for Lipschitz maps. Ann. Acad. Sci. Fenn. Ser. A I Math. 5, 49-72 (1980) Zbl 0411.57015 MR 0595177

[69] Väisälä, J.: Homeomorphisms of bounded length distortion. Ann. Acad. Sci. Fenn. Ser. A I Math. 12, 303-312 (1987) Zbl 0653.30009 MR 0951979

[70] Whyburn, G. T.: Analytic Topology. Amer. Math. Soc., Providence, RI (1963) Zbl 0117.15804 MR 0182943

[71] Youngs, J. W. T.: The topological theory of Fréchet surfaces. Ann. of Math. (2) 45, 753-785 (1944) Zbl 0061.10903 MR 0012222

[72] Youngs, J. W. T.: Homeomorphic approximations to monotone mappings. Duke Math. J. 15, 87-94 (1948) Zbl 0030.41603 MR 0024623 